\title{
Small-time versions of Strassen's law for Lévy processes
}

\author{
R. A. Maller
}

\section{ABSTRACT}

We study aspects of the 'small-time' behaviour (as $t \downarrow 0$ ) of a Lévy process $X(t)$, obtaining a very general small-time version of Strassen's almost sure (a.s.) functional law of the iterated logarithm (LIL) for random walks. The class of Lévy processes for which this holds is characterised by an explicit analytic condition on the Lévy measure of $X$, related to an analogous condition of Kesten for a generalised (large-time) random walk LIL. Both centred and uncentred versions of the small-time result are proved. Subsidiary results concerning functional weak convergence of $X(t)$ to Brownian motion as $t \downarrow 0$ are shown to be equivalent to the main a.s. results. The quadratic variation process of $X$ is considered, and applications via continuous functionals are suggested.

\section{Introduction and results}

Let $(X(t))_{t \geqslant 0}$ be a Lévy process on a probability space $(\Omega, \mathscr{F}, P)$ with $X(0)=0$ and canonical exponent $\Psi(\theta)$. Thus $E e^{i \theta X(t)}=e^{-t \Psi(\theta)}, \theta \in \mathbb{R}, t>0$, with

$$
\Psi(\theta)=-i \gamma \theta+\frac{\sigma^{2} \theta^{2}}{2}+\int_{\mathbb{R} \backslash\{0\}}\left(1-e^{i \theta x}+i \theta x 1_{\{|x| \leqslant 1\}}\right) \Pi(d x),
$$

where $\gamma \in \mathbb{R}, \sigma^{2} \geqslant 0$, and $\Pi(\cdot)$ is a measure on the Borel subsets of $\mathbb{R} \backslash\{0\}$ satisfying $\int_{\mathbb{R} \backslash\{0\}}\left(x^{2} \wedge 1\right) \Pi(d x)<\infty$. See, for example, Bertoin [2] and Sato [16] for basic properties of Lévy processes.

The local or small-time behaviour of Lévy processes, that is, as $t \downarrow 0$, often viewed as a generalisation of some of the path properties of Brownian motion to a process with jumps, has long been a source of inspiration to probabilists, dating back to the time of Lévy himself, and many deep and subtle results have been obtained. With an upsurge in interest in Lévy processes in various application areas, especially in physics and finance recently, this kind of behaviour has assumed enhanced importance.

Our current investigation was motivated in part by a recent result of Bertoin et al. [3], who gave conditions under which a Lévy process satisfies

$$
-c=\liminf _{t \downarrow 0} \frac{X(t)}{\sqrt{t}}<\limsup _{t \downarrow 0} \frac{X(t)}{\sqrt{t}}=c, \text { a.s. }
$$

for a constant $c \in(0, \infty)$. It has previously been observed that small-time Lévy process results can often be conjectured from corresponding large-time results; the latter, in turn, often correspond exactly to analogous asymptotic results for random walks. Recent papers exploiting this kind of transference principle are, for example, $[\mathbf{8}, \mathbf{9}]$ (see also $[\mathbf{7}]$ for a recent review); and of course there are examples in the older literature too, cf. various results in $[\mathbf{2}]$ and [16]. Equation (1.2) is remarkable, however, in that it has no 'large-time' analogue; any nondegenerate Lévy process satisfies $\lim \sup _{t \uparrow \infty}|X(t)| / \sqrt{t}=+\infty$, a.s.

Received 27 March 2007; revised 30 January 2008; published online 7 October 2008.

2000 Mathematics Subject Classification 60G51, 60F15, 60F17, 60F05 (primary), s60J65, 60J75 (secondary). This research was partially supported by ARC grant DP0664603. 
Equation (1.2) has recently been generalised by Savov [17], who replaced the $\sqrt{t}$ norming with a function regularly varying with index $1 / 2$ at zero, and by Buchmann et al. [5], who gave a functional version for (1.2) in the spirit of Strassen's [18] functional law for random walks. Here we generalise both these results by developing theorems analogous to (1.2) in which the $\sqrt{t}$ norming is replaced by a quite general one (and centring of the process is allowed), and furthermore we give a functional law analogous to that of [5], for this more general iterated logarithm-like behaviour, as $t \downarrow 0$. These investigations yield very general small-time versions of Strassen's [18] functional law, for Lévy processes, and open the way to various interesting applications such as those in [5]. Despite the apparently idiosyncratic nature of (1.2), we will show that an analogue of a random-walk result of Kesten [12] provides the key to characterising this kind of behaviour, and that, analogous to the random-walk case, the governing principle is that the Lévy process needs to be attracted to normality, or relatively stable, at small times, at least through a subsequence. Except for Brownian motion and some other diffusions (see $[\mathbf{1}, \mathbf{6}, \mathbf{1 1}]$ ), this seems to be the first time these kinds of problems have been considered in a continuous time context.

We consider the family of processes

$$
\left(\frac{X(t y)-t y a(t)}{b(t)}\right)_{0 \leqslant y \leqslant 1}
$$

indexed by $t, t>0$, where $a(\cdot)$ and $b(\cdot)>0$ are nonstochastic functions, to be chosen appropriately. It turns out that the centring function $a(t)$ can be specified in terms of the truncated mean function $\nu(\cdot)$ defined by

$$
\nu(x)=\gamma-\int_{x<|y| \leqslant 1} y \Pi(d y), \quad x>0,
$$

while the norming function $b(t)$ is related to the truncated variance function

$$
V(x)=\sigma^{2}+\int_{0<|y| \leqslant x} y^{2} \Pi(d y), \quad x>0 .
$$

For $x>0$, let $\bar{\Pi}^{+}(x)=\Pi\{(x, \infty)\}$ and $\bar{\Pi}^{-}(x)=\Pi\{(-\infty,-x)\}$ be the positive and negative tails of $\Pi(\cdot)$, and define the tail sum $\bar{\Pi}(x)$ and tail difference $D(x)$ as

$$
\bar{\Pi}(x)=\bar{\Pi}^{+}(x)+\bar{\Pi}^{-}(x) \text { and } \quad D(x)=\bar{\Pi}^{+}(x)-\bar{\Pi}^{-}(x) .
$$

We will also use the following variants of $\nu$ and $V$ (defined for $x>0$ ):

$$
A(x)=\gamma+D(1)-\int_{x}^{1} D(y) d y=\nu(x)+x D(x),
$$

and

$$
U(x)=\sigma^{2}+2 \int_{0}^{x} y \bar{\Pi}(y) d y=V(x)+x^{2} \bar{\Pi}(x) .
$$

All functions, $\nu, V, A$, and $U$ are finite for each $x>0$ by virtue of properties of $\Pi(\cdot)$. It is easy to check also that $\lim _{x \downarrow 0} x \nu(x)=0=\lim _{x \downarrow 0} x A(x)$.

For each $t>0$, the process in $(1.3)$ is a member of $D_{0}[0,1]$, the càdlàg functions on $[0,1]$ which are 0 at 0 . Denote by $\mathscr{K}$ the class of absolutely continuous functions $f$ on $[0,1]$ with $f(0)=0$ and $\int_{0}^{1}\left(f^{\prime}(y)\right)^{2} d y \leqslant 1$. Now, $\mathscr{K}$ is a subset of $C_{0}[0,1]$, the continuous functions on $[0,1]$, which are 0 at 0 .

Our first theorem gives the general functional law for $X(t)$ at 0 , showing its equivalence to a kind of partial attraction (along a subsequence) of the process to normality at time 0 . Theorem 1 allows $X$ to be centred. Theorems 2 and 3 treat uncentred cases. Theorem 4 gives a further subsequential result related to the stability of $X$ at 0 , and Theorem 5 applies the results to the quadratic variation process of $X$. All proofs are given in Section 2. We will assume 
throughout that $\bar{\Pi}(0+)>0$, so that $X$ has a nontrivial jump component. The alternate case will be discussed separately (see Remark 1(i) below).

THEOREM 1. The following are equivalent:

(i)

$$
\limsup _{x \downarrow 0} \frac{V(x)}{x^{2} \bar{\Pi}(x)}=\infty
$$

(ii) there is a nonstochastic function $b(t)>0$ on $[0,1]$ such that the processes

$$
\left(\frac{X(t y)-t y \nu(b(t))}{b(t)}\right)_{0 \leqslant y \leqslant 1}
$$

have as their a.s. cluster set (as $t \downarrow 0)$ the class $\mathscr{K}$;

(iii) there is a nonstochastic sequence $t_{i} \downarrow 0$ and function $c(t) \downarrow 0$ such that, as $i \rightarrow \infty$, the processes

$$
\left(\frac{X\left(t_{i} y\right)-t_{i} y \nu\left(c\left(t_{i}\right)\right)}{c\left(t_{i}\right)}\right)_{0 \leqslant y \leqslant 1} \stackrel{W}{\Longrightarrow}(W(y))_{0 \leqslant y \leqslant 1} ;
$$

that is, converge weakly in $D_{0}[0,1]$, with the Skorokhod metric, to a standard Brownian motion $W(\cdot)$.

In (1.9), given an $\varepsilon_{0}>0$, it is possible to choose the function $b(t)$, depending on $\varepsilon$, so that $t^{-1 / 2+\varepsilon} b(t)$ is nondecreasing in $t \in[0,1]$ for each $\varepsilon \in\left(0, \varepsilon_{0}\right)$; in particular, $b(t)$ is then strictly increasing in $t \in[0,1]$.

REMARK 1. (i) To be precise, what is shown in the proof of Theorem 1 is the following. Let $\left(Z_{t}(y)\right)_{0 \leqslant y \leqslant 1}$ denote the process in (1.9), indexed by $t>0$. Then, under (1.8), there exists an $\widetilde{\Omega} \in \mathscr{F}$ with $P(\widetilde{\Omega})=1$ such that, for all $\omega \in \widetilde{\Omega}$,

$$
\lim _{t \downarrow 0} \inf _{g \in \mathscr{K}}\left\|Z_{t}(y, \omega)-g\right\|=0, \text { and } \liminf _{t \downarrow 0}\left\|Z_{t}(y, \omega)-f\right\|=0 \quad \text { for all } f \in \mathscr{K},
$$

where $\|\cdot\|$ denotes the uniform norm, $\|g\|=\sup _{0 \leqslant y \leqslant 1}|g(y)|$, of a function on $[0,1]$. The first part of (1.11) says that the uniform distance between function $Z_{t}$ and set $\mathscr{K}$ tends to 0 as $t \downarrow 0$; thus, all accumulation points of $\left(Z_{t}\right)_{t \geqslant 0}$ are in $\mathscr{K}$. The second part of (1.11) says that any $f$ in $\mathscr{K}$ is obtained as the limit (in norm) of a subsequence of $\left(Z_{t}\right)_{t \geqslant 0}$. We describe this, as in Theorem 1 and below, by saying that $\mathscr{K}$ is the a.s. cluster set of $\left(Z_{t}\right)_{t \geqslant 0}$. A rigorous development of the concepts required here, to properly take into account the nonseparability of the space $D_{0}[0,1]$, and convergence through the continuous time variable $t$, is in $[\mathbf{5}]$. We assume this background and proceed by proving (1.11), and an analogous version for Theorem 2 below.

(ii) The Brownian case: We excluded throughout, and in Theorem 1 , the case $\Pi(\cdot) \equiv 0$. However, suppose that this is so, and that $\sigma^{2}>0$. Then $X(t)=\gamma t+\sigma B(t)$ is a nontrivial Brownian motion. Part (ii) of Theorem 1 remains true in this case, with no further assumptions, and with the specific choices $b(t)=\sigma \ell(t)$, where $\ell(t):=\sqrt{2 t \log |\log t|}$, and $\nu(b(t))=\gamma$, for $t \in(0,1)$. This result is a small-time version of Strassen's [18] classic functional law of the iterated logarithm (LIL) for Brownian motion, and a proof of it is outlined in a remark following the proof of Proposition 1, in Section 2 below. It can be compared with a result of Gantert [11], who uses a time-inversion argument to transfer Strassen's large-time functional theorem for Brownian motion to the small-time setting; but she obtains only a sequential LIL at zero, by this method. The methodology developed in [5], and here, is needed for the continuous time result.

Note that (1.10) is trivially true when $X$ is a nondegenerate Brownian motion, with $t_{i}$ replaced by the continuous variable $t, c\left(t_{i}\right)$ replaced by $\sigma \sqrt{t}$, and $\nu\left(c\left(t_{i}\right)\right)$ by $\gamma$. With the 
convention that (1.8) holds when $\Pi(\cdot) \equiv 0$ and $\sigma^{2}>0$ (so that $V(x) \equiv \sigma^{2}>0$ ), Theorem 1 in fact remains true in this case.

The more precise LIL $((1.9)$ with $b(t)=\ell(t))$ actually holds whenever $\sigma^{2}>0$ (even if the jump component is present). Khintchine [13] (cf. Sato [16, Proposition 47.11, p. 358]) showed that $\lim \sup _{t \downarrow 0}|X(t)| / \ell(t)=\sigma \geqslant 0$ a.s. (for any Lévy process); applying this to the jump component of $X$ alone shows that the component is $o(\ell(t))$ as $t \downarrow 0$ and hence can be neglected when the Brownian component is present. In other words, when $\sigma^{2}>0$, regardless of $\Pi(\cdot)$, Part (ii) of Theorem 1 holds with $b(t)=\sigma \ell(t)$ and $\nu(b(t))=\gamma$. Further, Khintchine's [13] result supplies a converse: if for a Lévy process $X$, we have $\lim \sup _{t \downarrow 0}|X(t)| / \ell(t) \in(0, \infty)$ a.s., then necessarily $\sigma^{2}>0$.

(iii) The compound Poisson case: This case is also excluded, implicitly, in Theorem 1. If $X$ is compound Poisson, then $\sigma^{2}=0$ and $\Pi$ is a finite measure, so $V(x)=\mathrm{o}\left(x^{2}\right)=\mathrm{o}\left(x^{2} \bar{\Pi}(x)\right)$ as $x \downarrow 0$, and (1.8) could not hold. Of course, for a compound Poisson, $X(t)=0$ for all small $t$, a.s., so (1.10) and Part (ii) of Theorem 1 cannot hold either and, similarly, the situations in Theorems $2-5$ are ruled out too. Thus, throughout the paper, only the 'infinite activity' case $\bar{\Pi}(0+)=\infty$ is really relevant, when $\sigma^{2}=0$.

(iv) When (1.8) holds with 'lim sup' replaced by 'lim', the sequence $t_{i}$ in (1.10) may be replaced by the continuous variable $t$ and $c\left(t_{i}\right)$ by a continuous function of $t$ (and similarly in (1.13) and (1.14) below). Such results extend the 'domain of attraction of the normal' results in $[8]$.

(v) Theorem 1 is an analogue and extension of [12, Theorem 6], for random walks. The large-value analogue of (1.8) is the condition for a random walk to be in the 'domain of partial attraction of the normal', for large times; see Lévy [14, p. 113]). When $\sigma^{2}=0$ and $\bar{\Pi}(0+)>0$, equivalent to $(1.8)$ is

$$
\liminf _{x \downarrow 0} \frac{\bar{\Pi}(\mu x)}{\bar{\Pi}(x)} \leqslant \mu^{-2} \quad \text { for every } \mu>1 .
$$

In fact if (1.8) does not hold, then there are constants $c>0$ and $x_{0}>0$ such that, for every $\mu>1, \bar{\Pi}(\mu x) / \bar{\Pi}(x) \geqslant c \mu^{c-2}$ for $x \leqslant x_{0}$. We omit the proof.

(v) We can choose a canonical measure such that $\bar{\Pi}(x)=1 /(x|\log x|)^{2}$ for small $x>0$, and set $\sigma^{2}=0$. Then $V(x) \sim 2 /|\log x|$ as $x \downarrow 0$ and (1.8) holds. In this example, it can be shown that $b(t) / \sqrt{t}$ is bounded above and below by functions which are slowly varying as $t \downarrow 0$.

Theorem 2. Conditions (i) and (ii) are equivalent, where:

(i)

$$
\limsup _{x \downarrow 0} \frac{V(x)}{x^{2} \bar{\Pi}(x)+x|A(x)|}=\infty ;
$$

(ii) there is a nonstochastic sequence $t_{i} \downarrow 0$ and function $c(t) \downarrow 0$ such that

$$
\left(\frac{X\left(t_{i} y\right)}{c\left(t_{i}\right)}\right)_{0 \leqslant y \leqslant 1} \stackrel{W}{\Longrightarrow}(W(y))_{0 \leqslant y \leqslant 1} \quad \text { in } D_{0}[0,1], \text { as } i \rightarrow \infty,
$$

where $W(\cdot)$ is a standard Brownian motion;

(iii) further, conditions (i) and (ii) imply that there is a nonstochastic function $b(t)>0$ on $[0,1]$ such that the processes

$$
\left(\frac{X(t y)}{b(t)}\right)_{0 \leqslant y \leqslant 1}
$$

have as their a.s. cluster set (as $t \downarrow 0)$ the class $\mathscr{K}$.

As in Theorem 1 , given an $\varepsilon_{0}>0$, it is possible to choose $b(t)$, depending on $\varepsilon$, so that $t^{-1 / 2+\varepsilon} b(t)$ is nondecreasing in $t \in[0,1]$ for each $\varepsilon \in\left(0, \varepsilon_{0}\right)$. 
REMARK 2. (i) As in Remark 1 , when $\bar{\Pi}(\cdot) \equiv 0$ but $\sigma^{2}>0$ then $X(t y) / \sqrt{t}=(\gamma t+$ $\sigma B(t y)) / \sqrt{t} \stackrel{\mathrm{W}}{\Longrightarrow} \sigma B(y)$, as $t \downarrow 0$, so (1.14) holds with $t_{i}=t$ and $c(t)=\sigma \sqrt{t}$. Also, Part (iii) of Theorem 2 holds with $b(t)=\sigma \ell(t)=\sigma \sqrt{2 t \log |\log t|}$; the centring function $\operatorname{ty\nu }(b(t))=t y \gamma$ is o $(\ell(t))$ as $t \downarrow 0$, so can be deleted here. Equation (1.13) holds trivially, as $V(x) \equiv \sigma^{2}>0$ and $A(x) \equiv \gamma \in(-\infty, \infty)$.

(ii) In general it appears that Part (iii) of Theorem 2 does not imply (1.13); at any rate, it is possible to have $\lim \sup _{t \downarrow 0}|X(t)| / b(t)=1$ a.s., without (1.13) being true. Our next theorem addresses this issue.

THEOREM 3. The following are equivalent:

(i) There is a nonstochastic function $b(t)>0$ on $[0,1]$ such that

$$
\limsup _{t \downarrow 0} \frac{|X(t)|}{b(t)}=1 \text { a.s.; }
$$

(ii)

$$
\limsup _{x \downarrow 0} \frac{U(x)}{x^{2} \bar{\Pi}(x)}=\infty
$$

or

$$
\limsup _{x \downarrow 0} \frac{|A(x)|}{x \bar{\Pi}(x)}=\infty
$$

(iii)

$$
\limsup _{x \downarrow 0} \frac{U(x)+x|A(x)|}{x^{2} \bar{\Pi}(x)}=\infty ;
$$

(iv)

$$
\limsup _{x \downarrow 0} \frac{U(x)}{x^{2} \bar{\Pi}(x)+x|A(x)|}=\infty
$$

or

$$
\limsup _{x \downarrow 0} \frac{x|A(x)|}{U(x)}=\infty .
$$

As in Theorems 1 and 2 , it is possible to choose $b(t)$ so that $t^{-1 / 2+\varepsilon} b(t)$ is nondecreasing in $t \in[0,1]$ for each $0<\varepsilon<$ some $\varepsilon_{0}$.

REMARK 3. (i) Theorem 3 remains true with appropriate conventions regarding (1.17)(1.21) when $\bar{\Pi}(\cdot) \equiv 0$ but $\sigma^{2}>0$ or $\gamma \neq 0$. If $\bar{\Pi}(\cdot) \equiv 0$ and $\sigma^{2}>0$, then $X(t)=\gamma t+\sigma B(t)$ is a Brownian motion, so (1.16) holds with $b(t)=\sigma \ell(t)=\sigma \sqrt{2 t \log |\log t|}$ (regardless of the value of $\gamma$ ). If $\bar{\Pi}(\cdot) \equiv 0, \sigma^{2}=0$, and $\gamma \neq 0$, then $X(t)=\gamma t$, so (1.16) holds with $b(t)=|\gamma| t$.

(ii) We make an effort in the proofs below to define the norming sequences $b(t)$ in Theorems $1-3$ to be reasonably regular. In each case $b(t)$ is strictly increasing in $t$ and satisfies $\liminf _{t \downarrow 0} b(\mu t) / b(t)>\mu^{\delta}$, for some $\delta>0$, for each $\mu>1$. This property can be very useful in applications.

Equation (1.20) is equivalent to (1.13), so (1.15) of Theorem 2 implies (1.16) of Theorem 3, as it should, of course. Next we characterise condition (1.21). 
THEOREM 4. The following are equivalent:

(i) there is a nonstochastic sequence $t_{i} \downarrow 0$ and function $b(t) \downarrow 0$, such that, as $i \rightarrow \infty$,

$$
\frac{X\left(t_{i}\right)}{b\left(t_{i}\right)} \stackrel{\mathrm{P}}{\longrightarrow} \pm 1
$$

(ii)

$$
\limsup _{x \downarrow 0} \frac{|A(x)|}{\sqrt{\bar{\Pi}(x) U(x)}}=\infty ;
$$

(iii) Condition (1.21) holds.

Further, each of these implies $\sigma^{2}=0$.

REMARK 4. (i) Equation (1.22) is a kind of 'subsequential relative stability' of $X$ at 0 . For a continuous variable version, see $[\mathbf{8}$, Theorem 2.2]. If $\bar{\Pi}(0+)=0$, then $(1.22)$ holds if and only if $\sigma^{2}=0$ and $\gamma \neq 0$, in which case $X(t) /(t|\gamma|)= \pm 1$; see the remark following the proof of Theorem 4, in Section 2, below.

(ii) Taking into account Theorem 4, we see that Theorem 3 is a combination of generalised central limit behaviour, and a kind of generalised relative stability (weak law) behaviour, both as $t \downarrow 0$.

When a central limit behaviour obtains, under (1.17), we have (1.9) (because (1.17) implies (1.8)), and hence, as an immediate consequence, we have $\lim \sup _{t \downarrow 0}|X(t)-t \nu(b(t))| / b(t)=1$ a.s. The way this connects with (1.16) is, roughly speaking, that the centring function $t \nu(b(t))$ may be of order $b(t)$, in which case it can be discarded, or it may dominate $b(t)$ (through a subsequence), in which case it can be used to define a new norming function replacing $b(t)$. This rough intuition is formalised in the proof of Theorem 3.

Alternatively, generalised stability may obtain, under (1.18). As an aid to intuition, the situation simplifies considerably when $X$ is a subordinator, possibly with drift $\delta$; so assume this for the moment. Then $\bar{\Pi}^{-} \equiv 0$ and $A(x)=\delta+\int_{0}^{x} \bar{\Pi}^{+}(y) d y$. For a subordinator, $\lim _{t \downarrow} X(t) / t$ always exists and is finite, a.s. [8, Lemma 4.1], but may be 0 , and we can ask for the existence of a norming sequence such that $\lim _{\sup _{t \downarrow 0}} X(t) / b(t)=1$ a.s., or even $\lim _{t \downarrow 0} X(t) / b(t)=1$ a.s. Theorem 4.2 in [8] shows that the latter can occur only if the drift is positive. However, the former can occur more generally, as shown by our Theorem 3, when (1.18) holds. In the driftless subordinator case, (1.18) is equivalent to requiring that $A(x)$ not have 'positive increase' at 0 ([2, p. 87 and Example 7, p. 100]). Then Theorem 3 can be seen as an alternative to $[\mathbf{2}$, Proposition 10, p. 87], where it is shown that, if $A(x)$ does have positive increase at 0 , then $\lim \sup _{t \downarrow 0} X(t) / b(t)$ can take only the values 0 or $+\infty$, a.s., for any nondecreasing $b(t)$.

We remark here that, for a general Lévy process, (1.16) with 'lim' can hold only when $X$ is of bounded variation (bv) with non-zero drift; the proof of this is similar to that of $[\mathbf{8}$, Theorem 4.2], and reduces to it in the subordinator case.

(iii) In this paper we restricted ourselves to the 'small-time' functional behaviour of the Lévy process, since this seems not to have been analysed before and possesses the distinctive features mentioned in the introduction. We expect that there are similar 'large-time' versions of our results, that is, as $t \rightarrow \infty$, which closely parallel known random-walk functional limit theorems, and can be proved in a more routine manner.

Many applications of weak and strong functional limit theorems for random walks have been made and published in various areas, which we do not attempt to list here. These are 'largetime' applications, for which 'large-time' versions of our Lévy process results, as mentioned in the previous paragraph, would be relevant. Similar 'small-time' applications are certainly of interest. For some background on useful functionals see, for example, Bingham [4] and Strassen [18]. Buchmann et al. [5] give new and simple characterisations of the cluster sets 
obtained under some common continuous functionals, such as the supremum process of $X$ and the process reflected in its supremum.

We conclude with an application to the quadratic variation process of $X$.

TheOrem 5. Let $[X]_{t}=\sigma^{2} t+\sum_{0<s \leqslant t}\left(X_{s}-X_{s-}\right)^{2}$ denote the quadratic variation process of $X$. The following are equivalent, and equivalent to (1.8):

(i) there is a nonstochastic function $b(t)>0$ on $[0,1]$ such that

$$
\limsup _{t \downarrow 0} \frac{[X]_{t}}{b(t)}=1 \text { a.s.; }
$$

(ii) there are a nonstochastic sequence $t_{i} \downarrow 0$ and a function $c(t) \downarrow 0$ such that, as $i \rightarrow \infty$,

$$
\frac{[X]_{t_{i}}}{c\left(t_{i}\right)} \stackrel{\mathrm{P}}{\longrightarrow} 1 \text {. }
$$

REMARK 5. Here $[X]_{t}$ is a subordinator, with $\lim _{t \downarrow 0}[X]_{t} / t=\sigma^{2}$. Thus when $\sigma^{2}>0$, equation (1.24) holds with $b(t)=\sigma^{2} t$ (and 'limsup' replaced by 'lim'). When $\sigma^{2}=0, b(t)$ will be $\mathrm{o}(t)$ as $t \downarrow 0$, when it exists.

\section{Proofs}

The following representation (cf. [8, Lemma 6.1]) will be useful:

$$
X(t)=t \nu(b)+\sigma B(t)+X^{(1)}(t, b)+X^{(2)}(t, b),
$$

where $t>0, b>0$, and the components on the right-hand side are independent processes with jumps smaller than or equal in magnitude to $b$ (for $X^{(1)}$ ) and bigger in magnitude than $b$ (for $\left.X^{(2)}\right)$. Here, $B(t)$ is a standard Brownian motion, $X^{(1)}$ has finite moments of all orders, and $E X^{(1)}=0$. It is easy to check that

$$
\operatorname{Var}\left(\sigma B(t)+X^{(1)}(t, b)\right)=t V(b)
$$

where $V$ is defined in (1.5). Let $\mathbb{N}=\{1,2, \ldots\}$.

Proof of Theorem 1. This will be immediate from the following Propositions, 1-3.

Proposition 1. Given (1.8), we can define $b(t)$ so that the family of processes in (1.9) has $\mathscr{K}$ as its a.s. cluster set as $t \downarrow 0$.

Proof. Let (1.8) hold. Since $\bar{\Pi}(0+)>0$, we have $V(x)>0$ on a neighbourhood of 0 and, for some $x_{k} \downarrow 0$,

$$
\zeta_{k}:=\frac{x_{k}^{2} \bar{\Pi}\left(x_{k}\right)}{V\left(x_{k}\right)} \rightarrow 0, \quad \text { as } k \uparrow \infty .
$$

By taking a further subsequence if necessary, we can assume $\zeta_{k} \downarrow 0$ and $\zeta_{k} \leqslant k^{-2 / \varepsilon}$, where $\varepsilon \in(0,1 / 32)$ is fixed throughout the proof. Again $\bar{\Pi}(0+)>0$, and so we have $\zeta_{k}^{1 / 4} / \bar{\Pi}\left(x_{k}\right) \rightarrow 0$ and can further assume

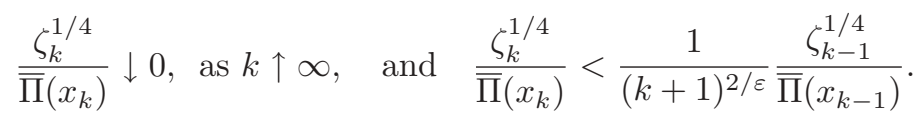


Take $x_{0}=1$ and (rescaling if necessary) $V(1) \wedge \bar{\Pi}(1)>0$. Then let $\zeta_{0}=\bar{\Pi}^{4}(1)$. Now define $b(t)$, depending on $\varepsilon$, on $[0,1]$, by: $b(0)=0$ and, for $k=1,2, \ldots$,

$$
b(t)=t^{(1 / 2)-\varepsilon}\left(\frac{\zeta_{k-1}^{1 / 4}}{\bar{\Pi}\left(x_{k-1}\right)}\right)^{\varepsilon} \sqrt{2 V\left(x_{k-1}\right) \log (k+1)}, \quad \text { when } \frac{\zeta_{k}^{1 / 4}}{\bar{\Pi}\left(x_{k}\right)}<t \leqslant \frac{\zeta_{k-1}^{1 / 4}}{\bar{\Pi}\left(x_{k-1}\right)} .
$$

(We use 'log' to denote log to base $e$, with a subscript if the base is otherwise.) Take $0<\lambda<1$ and define $r_{k}=r_{k}(\lambda), k=0,1, \ldots$, by

$$
r_{k}=\log _{\lambda}\left(\frac{\zeta_{k}^{1 / 4}}{\bar{\Pi}\left(x_{k}\right)}\right) \quad \text { so } \quad \frac{\zeta_{k}^{1 / 4}}{\bar{\Pi}\left(x_{k}\right)}=\lambda^{r_{k}}
$$

Then $r_{k} \uparrow \infty$, and by (2.4) we further have $r_{k}-r_{k-1}>-\log _{\lambda}(k+1)^{2 / \varepsilon} \rightarrow+\infty$.

Note that $b(t)$ does not depend on $\lambda$; nevertheless we have

$$
b(t)=t^{(1 / 2)-\varepsilon} \lambda^{\varepsilon r_{k-1}} \sqrt{2 V\left(x_{k-1}\right) \log (k+1)}, \quad \text { when } \lambda^{r_{k}}<t \leqslant \lambda^{r_{k-1}} .
$$

Now $b(t) / t^{(1 / 2)-\varepsilon}$ is constant on $\left(\lambda^{r_{k}}, \lambda^{r_{k-1}}\right]$, so nondecreasing on this interval, and thus is nondecreasing on $[0,1]$, because at a jump point

$$
\begin{aligned}
\frac{b^{2}\left(\lambda^{r_{k}}\right)}{b^{2}\left(\lambda^{r_{k}}+\right)} & =\lambda^{2 \varepsilon\left(r_{k}-r_{k-1}\right)} \frac{V\left(x_{k}\right) \log (k+2)}{V\left(x_{k-1}\right) \log (k+1)} \\
& \leqslant \frac{\log (k+2)}{(k+1)^{4} \log (k+1)} \quad\left(\text { since } x_{k} \downarrow \text { and } V\left(x_{k}\right) \downarrow \text { as } k \uparrow\right) \\
& <1, \quad \text { for } k=1,2, \ldots
\end{aligned}
$$

For integers $k$, let

$$
\begin{aligned}
& I_{k}=\left\{r \in \mathbb{N}: r_{k-1} \leqslant r \leqslant r_{k-1}+\varepsilon^{-1}\left|\log _{\lambda} k\right|\right\}, \\
& J_{k}=\left\{r \in \mathbb{N}: r_{k-1}+\varepsilon^{-1}\left|\log _{\lambda} k\right|<r<r_{k}\right\} .
\end{aligned}
$$

For $k$ large enough, $I_{k}$ is nonempty, and since we chose $r_{k}-r_{k-1}>\left|\log _{\lambda} k^{2 / \varepsilon}\right|$, the same is true of $J_{k}$. By omitting a finite number of terms we can thus assume that $I_{k}$ and $J_{k}$ are nonempty for $k=1,2, \ldots$.

Note that, with $c_{\lambda}=1-\lambda>0$,

$$
c_{\lambda} \sum_{k \geqslant 2} \sum_{r_{k-1} \leqslant r<r_{k}} \lambda^{r} \bar{\Pi}\left(x_{k-1}\right) \leqslant \sum_{k \geqslant 2} \lambda^{r_{k-1}} \bar{\Pi}\left(x_{k-1}\right)=\sum_{k \geqslant 2} \zeta_{k-1}^{1 / 4} \leqslant \sum_{k \geqslant 2} k^{-1 / 2 \varepsilon}<\infty
$$

and

$$
\begin{aligned}
\sum_{k \geqslant 1} \sum_{r \in J_{k}} \frac{\lambda^{r} V\left(x_{k-1}\right)}{b^{2}\left(\lambda^{r}\right)} & =\frac{1}{2} \sum_{k \geqslant 1} \sum_{r \in J_{k}} \frac{\lambda^{r} V\left(x_{k-1}\right)}{\lambda^{r-2 \varepsilon r} \lambda^{2 \varepsilon r_{k-1}} V\left(x_{k-1}\right) \log (k+1)} \\
& \leqslant \sum_{k \geqslant 1} \lambda^{-2 \varepsilon r_{k-1}} \sum_{r>r_{k-1}+\varepsilon^{-1}\left|\log _{\lambda} k\right|} \lambda^{2 \varepsilon r} \\
& \leqslant c_{\lambda}^{-1} \sum_{k \geqslant 1} \lambda^{-2 \log _{\lambda} k}<\infty .
\end{aligned}
$$

We further have

$$
\lim _{k \rightarrow \infty} \max _{r_{k-1} \leqslant r<r_{k}} \lambda^{r} \bar{\Pi}\left(x_{k-1}\right)=0 \quad \text { and } \quad \lim _{k \rightarrow \infty} \max _{r_{k-1} \leqslant r<r_{k}} \frac{\lambda^{r} V\left(x_{k-1}\right)}{b^{2}\left(\lambda^{r}\right)}=0 .
$$

The first of these follows from (2.7), and for the second, when $r_{k-1} \leqslant r<r_{k}$ we have $\lambda^{r_{k}}<$ $\lambda^{r} \leqslant \lambda^{r_{k-1}}$, and so by (2.6)

$$
b^{2}\left(\lambda^{r}\right)=2 \lambda^{r-2 \varepsilon r} \lambda^{2 \varepsilon r_{k-1}} V\left(x_{k-1}\right) \log (k+1) \geqslant 2 \lambda^{r} V\left(x_{k-1}\right) \log k .
$$


To complete the proof of Proposition 1, we need Lemmas 1-4, following. Let $p \geqslant 1$ be an integer and let $c_{1}, \ldots, c_{p}$ be constants such that $\sum_{1}^{p} c_{j}^{2}=1$. Define

$$
X_{p}(t)=\sum_{j=1}^{p} c_{j}(X(j t)-X((j-1) t)) .
$$

Let $c .=c_{1}+\ldots+c_{p}$, and set

$$
\Phi(x)=\frac{1}{\sqrt{2 \pi}} \int_{-\infty}^{x} e^{-z^{2} / 2} d z \quad \text { and } \quad \bar{\Phi}(x)=1-\Phi(x) .
$$

Recall the definitions of $\nu(\cdot), X^{(1)}, X^{(2)}$, in (1.4) and (2.1).

Lemma 1. If $y>0$ and $0<\lambda<1$, the series

$$
\sum_{k} \sum_{r \in I_{k}} \sup _{x \in \mathbb{R}}\left|P\left\{X_{p}\left(y \lambda^{r}\right)-c \cdot y \lambda^{r} \nu\left(x_{k-1}\right) \leqslant x \sqrt{y \lambda^{r} V\left(x_{k-1}\right)}\right\}-\Phi(x)\right|
$$

is finite for each $p \geqslant 1$ and, if $a>1$, then

$$
\sum_{r \geqslant 1} P\left\{\left|X_{p}\left(y \lambda^{r}\right)-c \cdot y \lambda^{r} \nu\left(b\left(\lambda^{r}\right)\right)\right|>a y^{1 / 2} b\left(\lambda^{r}\right)\right\}<\infty .
$$

Proof. Take $k \geqslant 1$ and $y>0$. Fix an integer $p \geqslant 1$. Define

$$
X_{p}^{(\ell)}\left(t, x_{k-1}\right)=\sum_{j=1}^{p} c_{j}\left(X^{(\ell)}\left(j t, x_{k-1}\right)-X^{(\ell)}\left((j-1) t, x_{k-1}\right)\right),
$$

for $\ell=1,2$. The summands on the right-hand side are independent for each $\ell=1,2$, and so also are they in

$$
B_{p}(t):=\sum_{j=1}^{p} c_{j}(B(j t)-B((j-1) t)) .
$$

Then, using (2.1) with $b=x_{k-1}$, we can write

$$
X_{p}(t)=\operatorname{c.t} \nu\left(x_{k-1}\right)+\sigma B_{p}(t)+X_{p}^{(1)}\left(t, x_{k-1}\right)+X_{p}^{(2)}\left(t, x_{k-1}\right) .
$$

Now

$$
\begin{aligned}
P\left(\sup _{0 \leqslant s \leqslant t}\left|X^{(2)}(s, b)\right|=0\right) & \geqslant P(\text { no jumps of magnitude }>b \text { occur by timet }) \\
& =e^{-t \bar{\Pi}(b)}
\end{aligned}
$$

so

$$
\begin{aligned}
& \sum_{k \geqslant 1} \sum_{r_{k-1} \leqslant r<r_{k}} P\left\{\sup _{0 \leqslant s \leqslant y \lambda^{r}}\left|X^{(2)}\left(s, x_{k-1}\right)\right| \neq 0\right\} \\
& \quad \leqslant \sum_{k \geqslant 1} \sum_{r_{k-1} \leqslant r<r_{k}}\left(1-e^{-y \lambda^{r} \bar{\Pi}\left(x_{k-1}\right)}\right) \leqslant y \sum_{k \geqslant 1} \sum_{r_{k-1} \leqslant r<r_{k}} \lambda^{r} \bar{\Pi}\left(x_{k-1}\right) .
\end{aligned}
$$

The last expression is finite by (2.7). Thus in verifying (2.12) we can ignore $X_{p}^{(2)}$ in (2.14) and just deal with $B_{p}$ and $X_{p}^{(1)}$. Take $r \in I_{k}$ and let

$$
\widetilde{X}=\frac{\sigma B_{p}\left(y \lambda^{r}\right)+X_{p}^{(1)}\left(y \lambda^{r}, x_{k-1}\right)}{\sqrt{y \lambda^{r} V\left(x_{k-1}\right)}} .
$$


This has expectation 0 , variance $\sum_{j=1}^{p} c_{j}^{2}=1$ (by (2.2)), and characteristic function

$$
E e^{i \theta \widetilde{X}}=\exp \left(-y \lambda^{r} \Psi^{(1)}\left(\theta / \sqrt{y \lambda^{r} V\left(x_{k-1}\right)}\right)\right)=\exp \left(-y \lambda^{r} \Psi^{(1)}\left(\theta^{\prime}\right)\right), \text { say }
$$

where $\Psi^{(1)}(\theta)$ satisfies

$$
e^{-t \Psi^{(1)}(\theta)}=E e^{i \theta\left(\sigma B_{p}(t)+X_{p}^{(1)}\left(t, x_{k-1}\right)\right)}=\prod_{j=1}^{p} E e^{i \theta c_{j}\left(\sigma B(t)+X^{(1)}\left(t, x_{k-1}\right)\right)},
$$

and $\theta^{\prime}=\theta / \sqrt{y \lambda^{r} V\left(x_{k-1}\right)}$. Thus we can write

$$
\begin{aligned}
\Psi^{(1)}(\theta) & =\frac{1}{2} \sigma^{2} \theta^{2}+\sum_{j=1}^{p} \int_{|x| \leqslant x_{k-1}}\left(1-e^{i \theta c_{j} x}+i \theta c_{j} x\right) \Pi(d x) \\
& =\frac{1}{2} \sigma^{2} \theta^{2}+\sum_{j=1}^{p} \int_{|x| \leqslant x_{k-1}}\left(\frac{1}{2} \theta^{2} c_{j}^{2} x^{2}+a\left(\theta c_{j} x\right) \theta^{3} x^{3}\right) \Pi(d x),
\end{aligned}
$$

where $\left|a\left(\theta c_{j} x\right)\right| \leqslant 5$ when $|\theta x| \leqslant 1$ (recall that $\sum_{j=1}^{p} c_{j}^{2}=1$, so $\left|c_{j}\right| \leqslant 1$ ). Substituting $\theta^{\prime}$ for $\theta$, and using the definition of $V(x)$ in (1.5), gives

$$
\begin{aligned}
\left|y \lambda^{r} \Psi^{(1)}\left(\theta^{\prime}\right)-\frac{1}{2} \theta^{2}\right| & \leqslant \frac{5 y \lambda^{r}|\theta|^{3}}{\left(y \lambda^{r} V\left(x_{k-1}\right)\right)^{3 / 2}} \int_{|x| \leqslant x_{k-1}}|x|^{3} \Pi(d x) \\
& \leqslant \frac{5 x_{k-1}|\theta|^{3}}{\sqrt{y \lambda^{r} V\left(x_{k-1}\right)}}
\end{aligned}
$$

provided that we keep $\left|\theta^{\prime} x_{k-1}\right|=\left|\theta x_{k-1}\right| / \sqrt{y \lambda^{r} V\left(x_{k-1}\right)} \leqslant 1$. Now $r \in I_{k}$, so $r \leqslant r_{k-1}+$ $\varepsilon^{-1}\left|\log _{\lambda} k\right|$, and we have

$$
\frac{x_{k-1}^{2}}{\lambda^{r} V\left(x_{k-1}\right)} \leqslant \frac{x_{k-1}^{2} k^{1 / \varepsilon}}{\lambda^{r_{k-1}} V\left(x_{k-1}\right)}=\frac{x_{k-1}^{2} \bar{\Pi}\left(x_{k-1}\right)}{V\left(x_{k-1}\right)} \frac{k^{1 / \varepsilon}}{\zeta_{k-1}^{1 / 4}}=\zeta_{k-1}^{3 / 4} k^{1 / \varepsilon} \leqslant k^{-1 /(2 \varepsilon)} .
$$

Hence

$$
\left|y \lambda^{r} \Psi^{(1)}\left(\theta^{\prime}\right)-\frac{1}{2} \theta^{2}\right| \leqslant 5|\theta|^{3} k^{-1 /(4 \varepsilon)} / \sqrt{y}
$$

and so

$$
\begin{aligned}
\left|E e^{i \theta \widetilde{X}}-e^{-(1 / 2) \theta^{2}}\right| & =e^{-(1 / 2) \theta^{2}}\left|e^{-y \lambda^{r} \Psi^{(1)}\left(\theta^{\prime}\right)+(1 / 2) \theta^{2}}-1\right| \\
& \leqslant 5 e^{-(1 / 2) \theta^{2}}|\theta|^{3} k^{-1 /(4 \varepsilon)} e^{5|\theta|^{3} k^{-1 /(4 \varepsilon)} / \sqrt{y}} / \sqrt{y}
\end{aligned}
$$

This is valid provided that $|\theta| \leqslant k^{1 /(4 \varepsilon)}$, since then

$$
\left|\theta^{\prime} x_{k-1}\right|=\left|\theta x_{k-1}\right| / \sqrt{y \lambda^{r} V\left(x_{k-1}\right)} \leqslant\left(k^{1 /(4 \varepsilon)}\right)\left(k^{-1 /(2 \varepsilon)}\right) / \sqrt{y} \leqslant 1
$$

for $k$ large enough, depending on $y$. In fact, keep $|\theta| \leqslant k^{1 /(16 \varepsilon)}:=\tau$, and get, for $c>0$,

$$
\begin{aligned}
& \int_{-\tau}^{\tau}\left|\theta^{-1}\left(E e^{i \theta \widetilde{X}}-e^{-(1 / 2) \theta^{2}}\right)\right| d \theta+c \tau^{-1} \\
& \quad \leqslant 5 y^{-1 / 2} k^{-1 /(4 \varepsilon)} \int_{-\tau}^{\tau} \theta^{2} e^{-(1 / 2) \theta^{2}+5|\theta|^{3} y^{-1 / 2} k^{-1 /(4 \varepsilon)}} d \theta+c k^{-1 /(16 \varepsilon)} \\
& \quad \leqslant \mathrm{O}\left(k^{-1 /(16 \varepsilon)}\right)=\mathrm{O}\left(k^{-2}\right) \quad(\text { since } 0<\varepsilon<1 / 32) .
\end{aligned}
$$

An application of Petrov [15, Theorem 2, p. 109] proves (2.12). 
Next, take $a>1$ and put $x=x(k, r)=a b\left(\lambda^{r}\right) / \sqrt{\lambda^{r} V\left(x_{k-1}\right)}$ in (2.12). For $r_{k-1} \leqslant r<r_{k}$ we have by $(2.10)$ that $x^{2}(k, r) \geqslant 2 a^{2} \log k$. Thus $x(k, r) \rightarrow \infty$ as $k \rightarrow \infty$ and hence, since $a>1$,

$$
\sum_{k} \sum_{r_{k-1} \leqslant r<r_{k}} \bar{\Phi}(x(k, r)) \leqslant c \sum_{k} \sum_{r_{k-1} \leqslant r<r_{k}} e^{-(1 / 2) x^{2}(k, r)} \leqslant c \sum_{k} \sum_{r_{k-1} \leqslant r<r_{k}} e^{-a^{2} \log k}
$$

converges ( $c$ denotes positive constants). Hence, from (2.12),

$$
\sum_{k} \sum_{r \in I_{k}} P\left\{\left|X_{p}\left(y \lambda^{r}\right)-c \cdot y \lambda^{r} \nu\left(x_{k-1}\right)\right|>a y^{(1 / 2)} b\left(\lambda^{r}\right)\right\}<\infty .
$$

We can replace $r \in I_{k}$ here by $r_{k-1} \leqslant r<r_{k}$, because of (2.16) and Chebychev's inequality:

$$
\begin{aligned}
& \sum_{k \geqslant 1} \sum_{r \in J_{k}} P\left\{\left|\sigma B_{p}\left(y \lambda^{r}\right)+X_{p}^{(1)}\left(y \lambda^{r}, x_{k-1}\right)\right|>a y^{(1 / 2)} b\left(\lambda^{r}\right)\right\} \\
& \quad \leqslant \sum_{k \geqslant 1} \sum_{r \in J_{k}} \frac{y \lambda^{r} V\left(x_{k-1}\right)}{a^{2} y b^{2}\left(\lambda^{r}\right)}<\infty \quad(\text { see }(2.8)) .
\end{aligned}
$$

Equation (2.18) then gives (2.13), if we replace $\nu\left(x_{k-1}\right)$ by $\nu\left(b\left(\lambda^{r}\right)\right)$ in it. We show

$$
\sup _{\lambda^{r} k<t \leqslant \lambda^{r_{k-1}}} \frac{t\left|\nu\left(x_{k-1}\right)-\nu(b(t))\right|}{b(t)} \rightarrow 0 \quad(k \rightarrow \infty) .
$$

Let $t \in\left(\lambda^{r_{k}}, \lambda^{r_{k-1}}\right]$. If $b(t) \geqslant x_{k-1}$ we have

$$
\frac{t\left|\nu\left(x_{k-1}\right)-\nu(b(t))\right|}{b(t)}=\frac{t\left|\int_{x_{k-1}<|x| \leqslant b(t)} x \Pi(d x)\right|}{b(t)} \leqslant \lambda^{r_{k-1}} \bar{\Pi}\left(x_{k-1}\right),
$$

and this tends to 0 as $k \rightarrow \infty$ by $(2.9)$. If $b(t)<x_{k-1}$ we have

$$
\frac{t\left|\nu\left(x_{k-1}\right)-\nu(b(t))\right|}{b(t)}=\frac{t\left|\int_{b(t)<|x| \leqslant x_{k-1}} x^{-1} x^{2} \Pi(d x)\right|}{b(t)} \leqslant \frac{t V\left(x_{k-1}\right)}{b^{2}(t)},
$$

and this tends to 0 , uniformly in $\lambda^{r_{k}}<t \leqslant \lambda^{r_{k-1}}$, as $k \rightarrow \infty$, again by (2.9). This proves (2.19), and (2.19) implies, as $k \rightarrow \infty$,

$$
\sup _{r_{k-1} \leqslant r<r_{k}} \frac{y \lambda^{r}\left|\nu\left(x_{k-1}\right)-\nu\left(b\left(\lambda^{r}\right)\right)\right|}{b\left(\lambda^{r}\right)} \leqslant \sup _{\lambda^{r} k<t \leqslant \lambda^{r_{k-1}}} \frac{t y\left|\nu\left(x_{k-1}\right)-\nu(b(t))\right|}{b(t)} \rightarrow 0 .
$$

Therefore, we can replace $\nu\left(x_{k-1}\right)$ by $\nu\left(b\left(\lambda^{r}\right)\right)$, as claimed, completing Lemma 1 .

LEMma 2. We have, for any $y>0$,

$$
\limsup _{t \downarrow 0} \sup _{0 \leqslant s \leqslant 1} \frac{\mid X_{p}(t s y)-\text { c.tsy }(b(t)) \mid}{b(t)} \leqslant \sqrt{y}, \quad \text { a.s. }
$$

Proof. We first prove (2.20) with $s=1$. Given $y>0, t \in(0,1)$, take $0<\lambda<1$, choose the integer $r(t, \lambda)$ such that $\lambda^{r}<t \leqslant \lambda^{r-1}$, and let $k=\min \left\{\ell: r_{\ell}>r\right\}$. Since $r_{j}-r_{j-1} \rightarrow \infty$ we then have $r_{k-1} \leqslant r-1<r \leqslant r_{k}$ for large $r$, thus $\lambda^{r_{k}} \leqslant \lambda^{r}<t \leqslant \lambda^{r-1} \leqslant \lambda^{r_{k-1}}$. As $t \downarrow 0$, $r_{k} \rightarrow \infty$, so

$$
\begin{aligned}
\frac{\left|X_{p}(t y)-c \cdot t y \nu(b(t))\right|}{b(t)} \leqslant & \frac{\left|X_{p}\left(y \lambda^{r}\right)-c \cdot y \lambda^{r} \nu\left(b\left(\lambda^{r}\right)\right)\right|}{b\left(\lambda^{r}\right)} \\
& +\frac{\sup _{\lambda^{r}<s \leqslant \lambda^{r-1}}\left|X_{p}(y s)-X_{p}\left(y \lambda^{r}\right)-c \cdot y\left(s-\lambda^{r}\right) \nu\left(b\left(\lambda^{r}\right)\right)\right|}{b\left(\lambda^{r}\right)}+\mathrm{o}(1) ;
\end{aligned}
$$


note that $t\left|\nu(b(t))-\nu\left(b\left(\lambda^{r}\right)\right)\right|=\mathrm{o}(b(t))$ by (2.19). As $t \downarrow 0, r \rightarrow \infty$, so by (2.13) the first term on the right-hand side of $(2.21)$ has limsup no larger than $\sqrt{y}$, a.s. We need to show that the second term is negligible. Now for $\lambda^{r} \leqslant s \leqslant \lambda^{r-1}$, consider

$$
\begin{aligned}
& \left|X_{p}(y s)-X_{p}\left(y \lambda^{r}\right)-c \cdot y\left(s-\lambda^{r}\right) \nu\left(b\left(\lambda^{r}\right)\right)\right| \\
& \quad=\mid \sum_{j=1}^{p} c_{j}[(X(j y s)-X((j-1) y s)) \\
& \quad-\left(X\left(j y \lambda^{r}\right)-X\left((j-1) y \lambda^{r}\right)-y\left(s-\lambda^{r}\right) \nu\left(b\left(\lambda^{r}\right)\right)\right] \mid \\
& \leqslant 2 p \max _{1 \leqslant j \leqslant p}\left|X(j y s)-X\left(j y \lambda^{r}\right)-j y\left(s-\lambda^{r}\right) \nu\left(b\left(\lambda^{r}\right)\right)\right| .
\end{aligned}
$$

We will proceed by finding an a.s. bound for

$$
\frac{\sup _{\lambda^{r} \leqslant s \leqslant \lambda^{r-1}}\left|X(j y s)-X\left(j y \lambda^{r}\right)-j y\left(s-\lambda^{r}\right) \nu\left(b\left(\lambda^{r}\right)\right)\right|}{b\left(\lambda^{r}\right)}
$$

when $j=1,2, \ldots, p$. Since

$$
\left(X(j y s)-X\left(j y \lambda^{r}\right)\right)_{s \geqslant \lambda^{r}} \stackrel{D}{=}(X(j y s))_{s \geqslant 0},
$$

we can equivalently bound $\sup _{0 \leqslant s \leqslant j \lambda^{r}\left(\lambda^{-1}-1\right) y} \mid X(s)-s \nu\left(b\left(\lambda^{r}\right) \mid / b\left(\lambda^{r}\right)\right.$. For this, let

$$
Y^{(1)}\left(t, x_{k-1}\right):=\sigma B(t)+X^{(1)}\left(t, x_{k-1}\right),
$$

and use a maximal inequality (for example, [8, Equation (5.4)]) to write, for $t>0, x>0$,

$$
P\left\{\sup _{0 \leqslant s \leqslant t}\left|Y^{(1)}\left(s, x_{k-1}\right)\right|>x\right\} \leqslant 2 P\left\{\left|Y^{(1)}\left(t, x_{k-1}\right)\right|>x-\sqrt{2 t V\left(x_{k-1}\right)}\right\} .
$$

Take $t=y \lambda^{r}, x=a y^{(1 / 2)} b\left(\lambda^{r}\right)$. Now $\inf _{r_{k-1} \leqslant r<r_{k}} b^{2}\left(\lambda^{r}\right) /\left(\lambda^{r} V\left(x_{k-1}\right)\right) \rightarrow \infty$ by (2.10), so for large $k, r_{k-1} \leqslant r<r_{k}$, and arbitrary $\delta \in(0, a)$, we get

$$
\begin{aligned}
& P\left\{\sup _{0 \leqslant s \leqslant y \lambda^{r}}\left|Y^{(1)}\left(s, x_{k-1}\right)\right|>a y^{(1 / 2)} b\left(\lambda^{r}\right)\right\} \\
& \quad \leqslant 2 P\left\{\left|Y^{(1)}\left(y \lambda^{r}, x_{k-1}\right)\right|>(a-\delta) y^{(1 / 2)} b\left(\lambda^{r}\right)\right\} .
\end{aligned}
$$

Hence from (2.13) (with $p=1=c_{1}$ ), together with (2.1), (2.16), and (2.19), we get, for all $a>1$,

$$
\sum_{r} P\left\{\sup _{0 \leqslant s \leqslant y \lambda^{r}}\left|X(s)-s \nu\left(b\left(\lambda^{r}\right)\right)\right|>a y^{\frac{1}{2}} b\left(\lambda^{r}\right)\right\}<\infty .
$$

Thus

$$
\limsup _{t \downarrow 0} \sup _{0 \leqslant s \leqslant j \lambda^{r}\left(\lambda^{-1}-1\right) y} \frac{\left|X_{p}(s)-c \cdot s \nu\left(b\left(\lambda^{r}\right)\right)\right|}{b\left(\lambda^{r}\right)} \leqslant \sqrt{j\left(\lambda^{-1}-1\right) y} \quad \text { a.s. }
$$

Hence the second term on the right-hand side of (2.21) has limsup no larger than $2 p \sqrt{p\left(\lambda^{-1}-1\right) y}$, a.s. Then let $\lambda \uparrow 1$ to obtain (2.20) with $s=1$. For $(2.20)$, argue as follows. Because (2.20) holds with $s=1$, given $\varepsilon>0$ we can find $t_{0}(\varepsilon) \in(0,1)$ such that, for all $0<t \leqslant t_{0}$,

$$
\frac{\left|X_{p}(t y)-c . t y \nu(b(t))\right|}{b(t)} \leqslant \sqrt{y}+\varepsilon, \text { a.s. }
$$

Thus, for all $0<t \leqslant t_{0}$,

$$
\sup _{0<s \leqslant t} \frac{\left|X_{p}(s y)-c . s y \nu(b(s))\right|}{b(s)} \leqslant \sqrt{y}+\varepsilon, \quad \text { a.s. }
$$


Replace $s$ by $s t$, note that $b(\cdot)$ increases, and use an argument similar to that in (2.19) to replace $\nu(b(s t))$ by $\nu(b(t))$, to get $(2.20)$ as stated.

Lemma 3. For each $y>0$ the process

$$
\frac{X_{p}(t y)-c \cdot t y \nu(b(t))}{b(t)}, \quad t \geqslant 0
$$

as a process in $\mathbb{R}$, has as its a.s. cluster set (as $t \downarrow 0)$ the interval $\left[-y^{1 / 2}, y^{1 / 2}\right]$.

Proof. Let $y>0,0<\lambda<1$, and $\beta_{k}=y \lambda^{r_{k-1}}$. In view of (2.20) it suffices to show

$$
P\left\{\left|\frac{X_{p}\left(\beta_{k}\right)-c \cdot \beta_{k} \nu\left(b\left(\lambda^{r_{k-1}}\right)\right)}{b\left(\lambda^{r_{k-1}}\right)}-b y^{1 / 2}\right|<\delta \text { i.o. as } k \rightarrow \infty\right\}=1
$$

for all $\delta>0,|b|<1$. Fix $\delta>0,|b|<1$. Choose $m \geqslant 2$ so that $m^{-1 / 2} \leqslant \delta$ and let $1 \leqslant q \leqslant m$. Now, with $\left.\Delta\left(q, m, j, \beta_{k}\right)=X\left((q / m+j-1) \beta_{k}\right)-X((q-1) / m+j-1) \beta_{k}\right)$ for $j=1,2, \ldots, p$, we have

$$
X_{p}\left(\beta_{k}\right)-c \cdot \beta_{k} \nu\left(b\left(\lambda^{r_{k-1}}\right)\right)=\sum_{q=1}^{m} \sum_{j=1}^{p} c_{j}\left(\Delta\left(q, m, j, \beta_{k}\right)-\left(\beta_{k} / m\right) \nu\left(b\left(\lambda^{r_{k-1}}\right)\right)\right) .
$$

To deal with the $q=1$ terms, note that $\Delta\left(q, m, j, \beta_{k}\right) \stackrel{D}{=} X\left(\beta_{k} / m\right)$, and

$$
\sum_{k \geqslant 1} P\left\{\left|X\left(\beta_{k} / m\right)-\left(\beta_{k} / m\right) \nu\left(b\left(\lambda^{r_{k-1}}\right)\right)\right|>a y^{1 / 2} b\left(\lambda^{r_{k-1}}\right) / m^{1 / 2}\right\}
$$

is finite for $a>1$ by (2.13) (take the case $p=1, c_{1}=1$ in (2.13)). Therefore

$$
\limsup _{k \rightarrow \infty} \frac{\left|X\left(\beta_{k} / m\right)-\left(\beta_{k} / m\right) \nu\left(b\left(\lambda^{r_{k-1}}\right)\right)\right|}{b\left(\lambda^{r_{k-1}}\right)} \leqslant \sqrt{\frac{y}{m}} \leqslant \frac{1}{\sqrt{m}} \text { a.s. }
$$

Thus for all large $k$, a.s. (noting that $\sum_{j=1}^{p}\left|c_{j}\right| \leqslant \sqrt{p}$ )

$$
\begin{aligned}
& \left|\frac{X_{p}\left(\beta_{k}\right)-c \cdot \beta_{k} \nu\left(b\left(\lambda^{r_{k-1}}\right)\right)}{b\left(\lambda^{r_{k-1}}\right)}-b y^{1 / 2}\right| \\
& \leqslant \sum_{q=2}^{m}\left|\frac{\sum_{j=1}^{p} c_{j}\left(\Delta\left(q, m, j, \beta_{k}\right)-\left(\beta_{k} / m\right) \nu\left(b\left(\lambda^{r_{k-1}}\right)\right)\right)}{b\left(\lambda^{r_{k-1}}\right)}-\frac{b y^{1 / 2}}{m-1}\right|+\sqrt{\frac{p}{m}} \\
& \leqslant m \bigvee_{q=2}^{m}\left|\frac{\sum_{j=1}^{p} c_{j}\left(\Delta\left(q, m, j, \beta_{k}\right)-\left(\beta_{k} / m\right) \nu\left(b\left(\lambda^{r_{k-1}}\right)\right)\right.}{b\left(\lambda^{r_{k-1}}\right)}-\frac{b y^{1 / 2}}{m-1}\right|+\sqrt{\frac{p}{m}},
\end{aligned}
$$

so it will suffice to show that $P\left(\cap_{q=2}^{m} A_{k}^{q}\right.$ i.o. in $\left.k\right)=1$, where $A_{k}^{q}$ is the event

$$
\left\{\left|\frac{\sum_{j=1}^{p} c_{j}\left(\Delta\left(q, m, j, \beta_{k}\right)-\left(\beta_{k} / m\right) \nu\left(b\left(\lambda^{r_{k-1}}\right)\right)\right)}{b\left(\lambda^{r_{k-1}}\right)}-\frac{b y^{1 / 2}}{m-1}\right| \leqslant \frac{\delta}{m-1}\right\} .
$$

Now keep $2 \leqslant q \leqslant m$. For $k$ large enough, $A_{k+1}^{q}$ is independent of $A_{k}^{q}$, because $A_{k}^{q}$ involves increments of $X(\cdot)$ only down as far as time

$$
((q-1) / m) \beta_{k} \geqslant \beta_{k} / m=y \lambda^{r_{k-1}} / m \quad(\text { since } q \geqslant 2),
$$

while $A_{k+1}^{q}$ involves $X(\cdot)$ only up as far as time

$$
(q / m+(p-1)) \beta_{k+1} \leqslant(1+(p-1)) \beta_{k+1}=p y \lambda^{r_{k}},
$$


and since $r_{k}-r_{k-1} \rightarrow \infty$ these are separated for large enough $k$. Hence by the Borel-Cantelli lemma, it suffices that

$$
\sum_{k} P\left(\bigcap_{q=2}^{m} A_{k}^{q}\right)=\infty
$$

However, for each $k, A_{k}^{2}, \ldots, A_{k}^{m}$ are independent, and so we need

$$
\sum_{k} \prod_{q=2}^{m} P\left(A_{k}^{q}\right)=\infty
$$

Now $A_{k}^{q}$ involves

$$
\begin{aligned}
\sum_{j=1}^{p} & c_{j}\left(X\left((q / m+j-1) \beta_{k}\right)-X\left(((q-1) / m+j-1) \beta_{k}\right)\right. \\
& \left.-\left(\beta_{k} / m\right) \nu\left(b\left(\lambda^{r_{k-1}}\right)\right)\right) \\
& \underline{D} \sum_{j=1}^{p} c_{j}\left(X\left(j \beta_{k} / m\right)-X\left((j-1) \beta_{k} / m\right)\right)-c \cdot\left(\beta_{k} / m\right) \nu\left(b\left(\lambda^{r_{k-1}}\right)\right) \\
= & X_{p}\left(\beta_{k} / m\right)-c .\left(\beta_{k} / m\right) \nu\left(b\left(\lambda^{r_{k-1}}\right)\right),
\end{aligned}
$$

so the sum in $(2.26)$ is

$$
\sum_{k \geqslant 1} P^{m-1}\left\{\left|\frac{X_{p}\left(\beta_{k} / m\right)-c \cdot\left(\beta_{k} / m\right) \nu\left(b\left(\lambda^{r_{k-1}}\right)\right)}{b\left(\lambda^{r_{k-1}}\right)}-\frac{b y^{1 / 2}}{m-1}\right| \leqslant \frac{\delta}{m-1}\right\} .
$$

Let

$$
\gamma_{k}=\frac{b\left(\lambda^{r_{k-1}}\right)}{\sqrt{y \lambda^{r_{k-1}} V\left(x_{k-1}\right) / m}}=\sqrt{2 m \log (k+1) / y} .
$$

By (2.12) of Lemma 1 we need the divergence of

$$
\sum_{k \geqslant 1}\left\{\Phi\left(\frac{\left(b y^{1 / 2}+\delta\right) \gamma_{k}}{m-1}\right)-\Phi\left(\frac{\left(b y^{1 / 2}-\delta\right) \gamma_{k}}{m-1}\right)\right\}^{m-1}
$$

and here the summands are bounded below by

$$
\begin{aligned}
& {\left[\frac{2 \delta \gamma_{k}}{m-1} \exp \left(-\frac{\left(b y^{1 / 2}+\delta\right)^{2} \gamma_{k}^{2}}{2(m-1)^{2}}\right)\right]^{m-1}} \\
& \quad \geqslant\left(\frac{2 \delta \sqrt{2 \log (k+1)}}{\sqrt{(m-1) y}}\right)^{m-1} \exp \left(-\frac{m\left(b y^{1 / 2}+\delta\right)^{2} \log (k+1)}{(m-1) y}\right) .
\end{aligned}
$$

These are the summands of a divergent series when $|b|<1, y \neq 0, \delta$ small enough, and $m$ large enough.

To prepare for Lemma 4 , let $\mathscr{L}_{p}$ be the continuous functions on $[0,1]$ which are linear on subintervals $[0,1 / p], \ldots,[(p-1) / p, 1]$, for $p=1,2, \ldots$. For $p=1,2, \ldots, t>0,0 \leqslant y \leqslant 1$, let $Z_{p}^{t}(y)$ be the linear interpolation of

$$
\begin{aligned}
\left(\frac{X(0)-(0) \nu(b(t))}{b(t)}, \frac{X(t / p)-(t / p) \nu(b(t))}{b(t)}, \ldots,\right. \\
\left.\frac{X((p-1) t / p)-t((p-1) / p) \nu(b(t))}{b(t)}, \frac{X(t)-t \nu(b(t))}{b(t)}\right)
\end{aligned}
$$


evaluated at $y$. Hence $Z_{p}^{t}(\cdot) \in \mathscr{L}_{p}$ for each $t>0, p=1,2, \ldots$ Following Finkelstein [10], let $f \in \mathscr{K}$ with $f_{p} \in \mathscr{L}_{p}$ its linear interpolation. Then ([10], p. 612)

$$
\sup _{0 \leqslant y \leqslant 1}\left|f(y)-f_{p}(y)\right| \leqslant 2 p^{-1 / 2} .
$$

Lemma 4. For each $p \geqslant 1,1 \leqslant i \leqslant p$,

$$
\limsup _{t \downarrow 0} \sup _{(i-1) / p \leqslant y \leqslant i / p}\left|\frac{X(t y)-t y \nu(b(t))}{b(t)}-Z_{p}^{t}(y)\right| \leqslant \frac{2}{p^{1 / 2}} \text { a.s. }
$$

Proof. The stochastic processes

$$
\left(D_{i}(t)\right)_{t \geqslant 0}:=\left(\sup _{(i-1) / p \leqslant y \leqslant i / p}|X(t y)-X((i-1) t / p)-t(y-(i-1) / p) \nu(b(t))|\right)_{t \geqslant 0}
$$

are i.i.d., $1 \leqslant i \leqslant p$, equal in distribution to

$$
\left(\sup _{0 \leqslant y \leqslant 1 / p}|X(t y)-t y \nu(b(t))|\right)_{t \geqslant 0} .
$$

Hence by $(2.20)$,

$$
\limsup _{t \downarrow 0}\left(\frac{D_{i}(t)}{b(t)}\right) \leqslant \frac{1}{p^{1 / 2}}, \quad \text { a.s. }
$$

Now for $(i-1) / p \leqslant y \leqslant i / p$ we have

$$
\begin{aligned}
Z_{p}^{t}(y)= & (p y-(i-1))(X(i t / p)-X((i-1) t / p)-(t / p) \nu(b(t))) / b(t) \\
& +(X((i-1) t / p)-((i-1) t / p) \nu(b(t))) / b(t),
\end{aligned}
$$

and hence (2.28) follows from

$$
\begin{aligned}
\left|\frac{X(t y)-t y \nu(b(t))}{b(t)}-Z_{p}^{t}(y)\right| & \leqslant \frac{|X(t y)-X((i-1) t / p)-(y-(i-1) / p) t \nu(b(t))|}{b(t)}+\frac{D_{i}(t)}{b(t)} \\
& \leqslant 2 \frac{D_{i}(t)}{b(t)} \quad \text { for }(i-1) / p \leqslant y \leqslant i / p .
\end{aligned}
$$

We can now complete the proof of Proposition 1. Define $\mathscr{V}: \mathscr{L}_{p} \mapsto \mathbb{R}^{p}$ by

$$
\mathscr{V}(f)=(f(1 / p), f(2 / p)-f(1 / p), \ldots, f(1)-f((p-1) / p),
$$

for $f \in \mathscr{L}_{p}$. Write $\nu_{t}$ temporarily for $\nu(b(t))$ and let $\mathbf{Z}_{t}$ be the $p$-vector

$$
\begin{aligned}
\left(\frac{X(t / p)-(t / p) \nu_{t}}{b(t)}, \frac{\left(X(2 t / p)-(2 t / p) \nu_{t}\right)-\left(X(t / p)-(t / p) \nu_{t}\right)}{b(t)}\right. & \\
\ldots, & \left.\frac{\left(X(t)-t \nu_{t}\right)-\left(X((p-1) t / p)-((p-1) t / p) \nu_{t}\right)}{b(t)}\right) .
\end{aligned}
$$

Take $\mathbf{c}=\left(c_{1}, \ldots, c_{p}\right) \in \mathbb{R}, c_{1}^{2}+c_{2}^{2}+\ldots+c_{p}^{2}=1$, and $c .=c_{1}+c_{2}+\ldots+c_{p}$. Then

$$
\mathbf{c}^{\prime} \mathbf{Z}_{t}=\frac{X_{p}(t / p)-c \cdot(t / p) \nu(b(t))}{b(t)},
$$

and so by Lemma $3, \mathscr{V}\left(Z_{p}^{t}\right)=\mathbf{Z}_{t}$ has as its a.s. cluster set (as $\left.t \downarrow 0\right) \mathscr{S}_{p}$, the sphere of radius $1 / \sqrt{p}$, in $\mathbb{R}^{p}$. Thus $Z_{p}^{t}(\cdot)$ has as its a.s. cluster set (as $\left.t \downarrow 0\right)$ the set $\mathscr{V}^{-1}\left(\mathscr{S}_{p}\right)=\mathscr{L}_{p} \cap \mathscr{K}$. Since $(X(t y)-t y \nu(b(t))) / b(t)$ is uniformly approximated by $Z_{p}^{t}(y)$ (by Lemma 4 ), and since $\mathscr{L}_{p} \cap \mathscr{K}$ is arbitrarily close to $\mathscr{K}$ for large $p$ (by (2.27)), we get the result for (1.9). 
REMARK 6. Our proof of Proposition 1 works with minor modification when $X_{t}=\gamma t+\sigma B_{t}$ is just a Brownian motion (and $\sigma^{2}>0$ ). Just take $b(t)=\sigma \ell(t)=\sigma \sqrt{2 t \log |\log t \log t|}$, and $\zeta_{k}=x_{k}^{2} / \sigma^{2}$ rather than as in (2.3). Then we still have $\zeta_{k} \rightarrow 0$ as $k \rightarrow \infty$, and we can work through the remainder of the proof simply omitting the $\bar{\Pi}(\cdot)$ when it occurs in expressions like (2.5).

Proposition 2. If the family of processes in (1.9) has $\mathscr{K}$ as its a.s. cluster set, for $t \downarrow 0$, then (1.8) holds.

Proposition 2 will follow immediately from the following lemma.

Lemma 5. Suppose that (1.8) fails, and that there are nonstochastic $a(t)$ and $b(t)(b(t)$ nondecreasing with $b(t)>0, b(0)=0)$ such that

$$
\limsup _{t \downarrow 0} \frac{|X(t)-a(t)|}{b(t)}<\infty \text { a.s. }
$$

Then

$$
\lim _{t \downarrow 0} \frac{X(t)-t \nu(b(t))}{b(t)}=0 \text { a.s. }
$$

Proof. Assume that (1.8) fails. Then it follows from (1.7) that $U(x) \leqslant c_{1} x^{2} \bar{\Pi}(x)$ for some $c_{1}>0$ for all small $x$. Since $\lim _{x \downarrow 0} x^{2} \bar{\Pi}(x)=0$, this means $\sigma^{2}=0$. Also, assume (2.29). By Blumenthal's $0-1$ law the left-hand side of (2.29) is a constant a.s., and so for some $c_{2}>0$

$$
\lim _{t \downarrow 0} P\left(|X(t)-a(t)|>2 c_{2} b(t)\right)=0 .
$$

By [8, Lemma 4.1] this means $t \bar{\Pi}\left(8 c_{2} b(t)\right) \rightarrow 0$ as $t \downarrow 0$. Take a subsequence $t_{j} \downarrow 0$ for which $t_{j} \bar{\Pi}\left(x b\left(t_{j}\right)\right) \rightarrow L(x)$, at points of continuity $x>0$ of $L$, for a nonincreasing function $L(\cdot)$. Then $L\left(8 c_{2}\right)=0$, thus $t_{j} U\left(8 c_{2} b\left(t_{j}\right)\right) / b^{2}\left(t_{j}\right) \rightarrow 0$, and so $t_{j} U\left(x b\left(t_{j}\right)\right) / b^{2}\left(t_{j}\right) \rightarrow 0$ for $0<x \leqslant 8 c_{2}$ as $t_{j} \downarrow 0$. However, $x^{2} \bar{\Pi}(x) \leqslant U(x)$ for all $x>0$, so $t_{j} \bar{\Pi}\left(x b\left(t_{j}\right)\right) \rightarrow 0$ for all $x>0$. Thus $t_{j} U\left(x b\left(t_{j}\right)\right) / b^{2}\left(t_{j}\right) \rightarrow 0$ for all $x>0$. Thus, as in the proof of [8, Theorem 3.1],

$$
\frac{X\left(t_{j}\right)-t_{j} \nu\left(b\left(t_{j}\right)\right)}{b\left(t_{j}\right)} \stackrel{P}{\longrightarrow} 0, \quad \text { as } t_{j} \downarrow 0
$$

where $\nu(x)$ is as in (1.4). Since this holds for all subsequences we have

$$
\frac{X(t)-t \nu(b(t))}{b(t)} \stackrel{P}{\longrightarrow} 0, \quad \text { as } t \downarrow 0 .
$$

A straightforward symmetrisation argument now shows that for (2.30) it suffices to assume $X(t)$ symmetric, so we can omit the centring function $a(t)$ in $(2.29)$. Then $(2.29)$ implies

$$
\sup _{2^{-n-1} \leqslant t<2^{-n}}\left|X(t)-X\left(2^{-n-1}\right)\right| \leqslant(c / 2) b\left(2^{-n}\right)
$$


(a.s., for $n$ large enough and some $c>0$ ), and the r.v.s. on the left-hand side are independent, $n=1,2, \ldots$, so by the Borel-Cantelli lemma,

$$
\begin{aligned}
\infty & >\sum_{n \geqslant 1} P\left(\sup _{2^{-n-1} \leqslant t<2^{-n}}\left|X(t)-X\left(2^{-n-1}\right)\right|>c b\left(2^{-n}\right)\right) \\
& =\sum_{n \geqslant 1} P\left(\sup _{0 \leqslant t<2^{-n-1}}|X(t)|>c b\left(2^{-n}\right)\right) \\
& \geqslant \sum_{n \geqslant 1} P\left(\sup _{0<t<2^{-n-1}}|\Delta X(t)|>2 c b\left(2^{-n}\right)\right),
\end{aligned}
$$

where $\Delta X(t)=X(t)-X(t-)$. Thus

$$
\begin{aligned}
\infty & >\sum_{n \geqslant 1}\left(1-P\left(\text { no jumps }>2 c b\left(2^{-n}\right) \text { in magnitude occur by time } 2^{-n-1}\right)\right) \\
& =\sum_{n \geqslant 1}\left(1-e^{-2^{-n-1} \bar{\Pi}\left(2 c b\left(2^{-n}\right)\right)}\right) .
\end{aligned}
$$

This forces $2^{-n} \bar{\Pi}\left(2 c b\left(2^{-n}\right)\right) \rightarrow 0$ and then $\sum 2^{-n} \bar{\Pi}\left(\varepsilon b\left(2^{-n}\right)\right)<\infty$ for $\varepsilon=2 c$. However $\bar{\Pi}(\mu x) \geqslant$ $c^{\prime} \bar{\Pi}(x)$ for some $c^{\prime}>0$, all small $x>0$, and all $\mu>1$ (see Remark 1 (v) following Theorem 1 ), so the series in fact converges for all $\varepsilon>0$. Hence also

$$
\sum_{n \geqslant 1} \frac{2^{-n} U\left(\varepsilon b\left(2^{-n}\right)\right)}{b^{2}\left(2^{-n}\right)}<\infty, \quad \text { for all } \varepsilon>0 .
$$

We will use this to show that

$$
\frac{\sup _{0<t \leqslant 2^{-n}}|X(t)|}{b\left(2^{-n-1}\right)} \rightarrow 0 \text { a.s. } \quad(n \rightarrow \infty)
$$

The proof of (2.30) in the symmetric case is then completed with a simple monotonicity argument. By the Borel-Cantelli lemma, for (2.32) it suffices that

$$
\sum_{n \geqslant 1} P\left\{\sup _{0 \leqslant t \leqslant 2^{-n}}|X(t)|>2 \varepsilon b\left(2^{-n-1}\right)\right\}<\infty, \quad \text { for all } \varepsilon>0 .
$$

For this it suffices by a maximal inequality (for example, [8, Equation (5.4)]), that

$$
\sum_{n \geqslant 1} P\left\{\left|X\left(2^{-n}\right)\right|>\varepsilon b\left(2^{-n-1}\right)\right\}<\infty, \quad \text { for all } \varepsilon>0 .
$$

Now (cf. (2.15))

$$
\begin{aligned}
\sum_{n \geqslant 1} P\left(X^{(2)}\left(2^{-n}, b\left(2^{-n-1}\right)\right) \neq 0\right) & \leqslant \sum_{n \geqslant 1}\left(1-e^{-2^{-n} \bar{\Pi}\left(b\left(2^{-n-1}\right)\right)}\right) \\
& \leqslant \sum_{n \geqslant 1} 2^{-n} \bar{\Pi}\left(b\left(2^{-n-1}\right)\right)<\infty,
\end{aligned}
$$

so by (2.1) (recall $\sigma^{2}=0$, and $\nu(\cdot)=0$, since $X$ is symmetric) it suffices that

$$
\sum_{n \geqslant 1} P\left(\left|X^{(1)}\left(2^{-n}, b\left(2^{-n-1}\right)\right)\right|>\varepsilon b\left(2^{-n-1}\right)\right\}<\infty
$$

for all $\varepsilon>0$. By Chebychev (see (2.2)) the last expression is not larger than

$$
\frac{1}{\varepsilon^{2}} \sum_{n \geqslant 1} \frac{2^{-n} V\left(b\left(2^{-n-1}\right)\right)}{b^{2}\left(2^{-n-1}\right)}
$$

and this is finite by $(2.31)$. 
Proposition 3. Equation (1.8) is equivalent to (1.10).

Proof. Let (1.8) hold, in which we take $U(x)$ instead of $V(x)$. Then choose $x_{i} \downarrow 0$ such that $\lim _{i \rightarrow \infty} U\left(x_{i}\right) / x_{i}^{2} \bar{\Pi}\left(x_{i}\right)=\infty$. Define

$$
t_{i}=\sqrt{\frac{x_{i}^{2}}{U\left(x_{i}\right) \bar{\Pi}\left(x_{i}\right)}}=\frac{1}{\bar{\Pi}\left(x_{i}\right)} \sqrt{\frac{x_{i}^{2} \bar{\Pi}\left(x_{i}\right)}{U\left(x_{i}\right)}} .
$$

Then $\lim _{i \rightarrow \infty} t_{i} \bar{\Pi}\left(x_{i}\right)=0$, and since $\bar{\Pi}(0+)>0$, also $\lim _{i \rightarrow \infty} t_{i}=0$. Let

$$
c_{i}^{2}=t_{i} U\left(x_{i}\right)=\frac{U\left(x_{i}\right)}{\bar{\Pi}\left(x_{i}\right)} \sqrt{\frac{x_{i}^{2} \bar{\Pi}\left(x_{i}\right)}{U\left(x_{i}\right)}} .
$$

Then $\lim _{i \rightarrow \infty} c_{i}=0$. On the other hand

$$
\frac{c_{i}^{2}}{x_{i}^{2}}=\frac{t_{i} U\left(x_{i}\right)}{x_{i}^{2}}=\sqrt{\frac{U\left(x_{i}\right)}{x_{i}^{2} \bar{\Pi}\left(x_{i}\right)}} \rightarrow \infty \quad(i \rightarrow \infty), \quad \text { by }(1.8) .
$$

Given $x>0$ choose $i$ so that $x c_{i} \geqslant x_{i}$. Then $t_{i} \bar{\Pi}\left(x c_{i}\right) \leqslant t_{i} \bar{\Pi}\left(x_{i}\right) \rightarrow 0$, and

$$
\begin{aligned}
\frac{t_{i} U\left(x c_{i}\right)}{c_{i}^{2}} & =1+\frac{t_{i}\left(U\left(x c_{i}\right)-U\left(x_{i}\right)\right)}{c_{i}^{2}}=1+\frac{2 t_{i} \int_{x_{i}}^{x c_{i}} y \bar{\Pi}(y) d y}{c_{i}^{2}} \\
& =1+\mathrm{O}\left(t_{i} \bar{\Pi}\left(x_{i}\right)\right)=1+\mathrm{o}(1) .
\end{aligned}
$$

Since $\lim _{i \rightarrow \infty} t_{i} \bar{\Pi}\left(x c_{i}\right)=0$, this means

$$
\lim _{i \rightarrow \infty} \frac{t_{i} V\left(x c_{i}\right)}{c_{i}^{2}}=1, \quad \text { for all } x>0 .
$$

Use (2.1) to write, for each $y>0$,

$$
X\left(t_{i} y\right)=t_{i} y \nu\left(c_{i}\right)+\sigma B\left(t_{i} y\right)+X^{(1)}\left(t_{i} y, c_{i}\right)+X^{(2)}\left(t_{i} y, c_{i}\right)
$$

By (2.15) we have $P\left(X^{(2)}\left(t_{i} y, c_{i}\right)=0\right) \geqslant e^{-t_{i} y \bar{\Pi}\left(c_{i}\right)} \rightarrow 1$, as $i \rightarrow \infty$, thus we have $X^{(2)}\left(t_{i} y, c_{i}\right) / c_{i} \stackrel{\mathrm{P}}{\longrightarrow} 0$ as $i \rightarrow \infty$. The characteristic function of $\left(\sigma B\left(t_{i} y\right)+X^{(1)}\left(t_{i} y, c_{i}\right)\right) / c_{i}$ is $\exp \left(-t_{i} y \Psi^{(1)}\left(\theta / c_{i}\right)\right)$, where

$$
\begin{aligned}
\Psi^{(1)}\left(\theta / c_{i}\right) & =\frac{\sigma^{2} \theta^{2}}{2 c_{i}^{2}}+\int_{|x| \leqslant c_{i}}\left(1-e^{i \theta x / c_{i}}+i \theta x / c_{i}\right) \Pi(d x) \\
& =\frac{\theta^{2}}{2 c_{i}^{2}}\left(\sigma^{2}+\int_{|x| \leqslant c_{i}} x^{2} \Pi(d x)\right)+\mathrm{O}\left(\frac{|\theta|^{3}}{c_{i}^{3}} \int_{|x| \leqslant c_{i}}|x|^{3} \Pi(d x)\right) \\
& =\frac{\theta^{2}}{2 c_{i}^{2}} V\left(c_{i}\right)+\mathrm{o}\left(t_{i}^{-1}\right) .
\end{aligned}
$$

The o $\left(t_{i}^{-1}\right)$ term follows by taking $0<\varepsilon<1$ and estimating

$$
\begin{aligned}
t_{i} c_{i}^{-3} \int_{|x| \leqslant c_{i}}|x|^{3} \Pi(d x) & \leqslant \varepsilon t_{i} c_{i}^{-2} \int_{|x| \leqslant \varepsilon c_{i}} x^{2} \Pi(d x)+t_{i} \bar{\Pi}\left(\varepsilon c_{i}\right) \\
& =\varepsilon t_{i} c_{i}^{-2} V\left(\varepsilon c_{i}\right)+o(1) \rightarrow \varepsilon, \text { as } i \rightarrow \infty
\end{aligned}
$$

Thus by (2.33)

$$
E e^{i \theta\left(\sigma B\left(t_{i} y\right)+X^{(1)}\left(t_{i} y, c_{i}\right)\right) / c_{i}} \rightarrow e^{-y \theta^{2} / 2},
$$


and so $\left(\sigma B\left(t_{i} y\right)+X^{(1)}\left(t_{i} y, c_{i}\right)\right) / c_{i} \stackrel{D}{\longrightarrow} N(0, y)$ as $t_{i} \downarrow 0$. Let $\left(Z_{i}(y)\right)_{0 \leqslant y \leqslant 1}$ denote the sequence of processes on the left-hand side of (1.10). Then by (2.34)

$$
Z_{i}(y)=\frac{X\left(t_{i} y\right)-t_{i} y \nu\left(c_{i}\right)}{c_{i}} \stackrel{D}{\longrightarrow} N(0, y), \quad \text { as } i \rightarrow \infty, \quad \text { for each } y>0 .
$$

To extend this to a functional version, we need to prove tightness. For $\varepsilon, \delta>0$,

$$
P\left(\sup _{0<y_{1}-y_{2} \leqslant \delta}\left|Z_{i}\left(y_{1}\right)-Z_{i}\left(y_{2}\right)\right|>\varepsilon\right)=P\left(\sup _{0<y \leqslant \delta}\left|X\left(t_{i} y\right)-t_{i} y \nu\left(c_{i}\right)\right|>\varepsilon c_{i}\right) .
$$

Again we will use (2.34), again discarding the $X^{(2)}$ component. Hence it suffices to consider $\sigma B\left(t_{i} y\right)+X^{(1)}\left(t_{i} y, c_{i}\right)$. For this,

$$
\begin{aligned}
P( & \left.\sup _{0 \leqslant y \leqslant \delta}\left|\sigma B\left(t_{i} y\right)+X^{(1)}\left(t_{i} y, c_{i}\right)\right|>\varepsilon c_{i}\right) \\
\quad= & \lim _{n \rightarrow \infty} P\left(\sup _{1 \leqslant j \leqslant n \delta}\left|\sum_{k=1}^{j} \Delta^{(1)}\left(k, t_{i} y, n, c_{i}\right)\right|>\varepsilon c_{i}\right),
\end{aligned}
$$

where

$$
\begin{aligned}
\Delta^{(1)}\left(k, t_{i} y, n, c_{i}\right)= & \sigma\left(B\left(k, t_{i} y / n\right)-B\left((k-1), t_{i} y / n\right)\right) \\
& +X^{(1)}\left(k t_{i} y / n, c_{i}\right)-X^{(1)}\left((k-1) t_{i} y / n, c_{i}\right)
\end{aligned}
$$

are i.i.d., $k=1,2, \ldots$, each with expectation 0 and variance $\left(t_{i} y / n\right) V\left(c_{i}\right)$, by $(2.2)$. By Kolmogorov's inequality, (2.37) is bounded by

$$
\limsup _{n \rightarrow \infty} \frac{n \delta\left(t_{i} y / n\right) V\left(c_{i}\right)}{\varepsilon^{2} c_{i}^{2}}=\frac{\delta t_{i} y V\left(c_{i}\right)}{\varepsilon^{2} c_{i}^{2}} .
$$

Thus, by (2.33), the lefthand side of (2.36) tends to 0 as $i \rightarrow \infty$; then $\delta \downarrow 0$, for each $\varepsilon>0$, which proves tightness.

Next we need convergence of finite-dimensional distributions, that is,

$$
\left(Z_{i}\left(y_{1}\right), Z_{i}\left(y_{2}\right), \ldots, Z_{i}\left(y_{p}\right)\right) \stackrel{D}{\longrightarrow}\left(W_{y_{1}}, W_{y_{2}}, \ldots, W_{y_{p}}\right)
$$

as $t_{i} \downarrow 0$, for any $0<y_{1}<y_{2}<\ldots<y_{p} \leqslant 1$, for all $p=1,2, \ldots$ This is true if and only if

$$
\begin{gathered}
\left(Z_{i}\left(y_{1}\right), Z_{i}\left(y_{2}\right)-Z_{i}\left(y_{1}\right), \ldots, Z_{i}\left(y_{p}\right)-Z_{i}\left(y_{p-1}\right)\right) \\
\stackrel{D}{\longrightarrow}\left(W_{y_{1}}, W_{y_{2}}-W_{y_{1}}, \ldots, W_{y_{p}}-W_{y_{p-1}}\right)
\end{gathered}
$$

where the right-hand side is a normal random vector with expectation 0 and covariance matrix $\operatorname{diag}\left(y_{1}, y_{1}-y_{2}, \ldots, y_{p}-y_{p-1}\right)$.

Let $a_{1}, a_{2}, \ldots, a_{p}$ be constants with $\sum a_{i}^{2}=1$. Consider (with $y_{0}=0$ )

$$
\sum_{k=1}^{p} a_{k}\left(Z_{i}\left(y_{k}\right)-Z_{i}\left(y_{k-1}\right)\right)=\frac{1}{c_{i}} \sum_{k=1}^{p} a_{k}\left(\Delta\left(t_{i}, y_{k}, y_{k-1}\right)-t_{i}\left(y_{k}-y_{k-1}\right) \nu\left(c_{i}\right)\right),
$$

where $\Delta\left(t_{i}, y_{k}, y_{k-1}\right)=X\left(t_{i} y_{k}\right)-X\left(t_{i} y_{k-1}\right)$, and $\left(\Delta\left(t_{i}, y_{k}, y_{k-1}\right)-t_{i}\left(y_{k}-y_{k-1}\right) \nu\left(c_{i}\right)\right) / c_{i}$ are independent, $1 \leqslant k \leqslant p$, with distributions the same as those of

$$
\left(X\left(t_{i}\left(y_{k}-y_{k-1}\right)\right)-t_{i}\left(y_{k}-y_{k-1}\right) \nu\left(c_{i}\right)\right) / c_{i}=Z_{i}\left(y_{k}-y_{k-1}\right) .
$$

By (2.35) each of these converges to $N\left(0, y_{k}-y_{k-1}\right)$. Thus

$$
\sum_{k=1}^{p} a_{k}\left(Z_{i}\left(y_{k}\right)-Z_{i}\left(y_{k-1}\right)\right) \stackrel{D}{\longrightarrow} N\left(0, \sum_{k=1}^{p} a_{k}^{2}\left(y_{k}-y_{k-1}\right)\right),
$$


where the variance is the same as that of $\sum_{k=1}^{p} a_{k}\left(W_{y_{k}}-W_{y_{k-1}}\right)$. Thus we have the required finite-dimensional convergence.

For the converse, suppose $\left(X\left(t_{i}\right)-a_{i}\right) / c_{i} \stackrel{D}{\longrightarrow} N(0,1)$ as $i \longrightarrow \infty$ for some $t_{i} \downarrow 0$ and nonstochastic $a_{i}, c_{i}$. By modifying the argument in the proof of $[\mathbf{8}$, Theorem 2.5] we get (1.8) immediately from

$$
\lim _{i \rightarrow \infty} t_{i} \bar{\Pi}\left(c_{i}\right)=0 \quad \text { and } \quad \liminf _{i \rightarrow \infty} \frac{t_{i} V\left(c_{i}\right)}{c_{i}^{2}}>0 .
$$

Proof of Theorem 2. As usual, assume $\bar{\Pi}(0+)>0$.

Proposition 4. Equation (1.13) is equivalent to (1.14).

Proof. Let (1.13) hold and choose $x_{k} \downarrow 0$ such that

$$
\frac{U\left(x_{k}\right)}{x_{k}^{2} \bar{\Pi}\left(x_{k}\right)} \geqslant \frac{V\left(x_{k}\right)}{x_{k}^{2} \bar{\Pi}\left(x_{k}\right)} \rightarrow \infty \quad \text { and } \quad \frac{U\left(x_{k}\right)}{x_{k}\left|A\left(x_{k}\right)\right|} \rightarrow \infty .
$$

Thus, $U\left(x_{k}\right)>0$ for large enough $k$. Define

$$
t_{k}=\min \left\{\sqrt{\frac{x_{k}^{2}}{\bar{\Pi}\left(x_{k}\right) U\left(x_{k}\right)}}, \sqrt{\frac{x_{k}^{3}}{\left|A\left(x_{k}\right)\right| U\left(x_{k}\right)}}\right\} .
$$

(If $A\left(x_{k}\right)=0$, then interpret the second component in (2.39) as $+\infty$.) Thus

$$
t_{k} \bar{\Pi}\left(x_{k}\right) \leqslant \sqrt{\frac{x_{k}^{2} \bar{\Pi}\left(x_{k}\right)}{U\left(x_{k}\right)}} \rightarrow 0,
$$

and since $\bar{\Pi}(0)>0$, we have $t_{k} \rightarrow 0$ as $k \rightarrow \infty$. Now let

$$
c_{k}^{2}=t_{k} U\left(x_{k}\right) \text {. }
$$

Then $c_{k} \rightarrow 0$ as $k \rightarrow \infty$. Also

$$
\frac{c_{k}^{2}}{x_{k}^{2}}=\min \left\{\sqrt{\frac{U\left(x_{k}\right)}{x_{k}^{2} \bar{\Pi}\left(x_{k}\right)}}, \sqrt{\frac{U\left(x_{k}\right)}{x_{k}\left|A\left(x_{k}\right)\right|}}\right\} \rightarrow \infty \quad(\text { by }(2.38)) .
$$

Thus $t_{k} \bar{\Pi}\left(x c_{k}\right) \rightarrow 0$ as $k \rightarrow \infty$ for all $x>0$. An easy calculation gives

$$
\lim _{k \rightarrow \infty} \frac{t_{k} U\left(x c_{k}\right)}{c_{k}^{2}}=1=\lim _{k \rightarrow \infty} \frac{t_{k} V\left(x c_{k}\right)}{c_{k}^{2}} \text { for all } x>0 .
$$

From this and $t_{k} \bar{\Pi}\left(x c_{k}\right) \rightarrow 0$ we obtain by a characteristic function argument

$$
\frac{X\left(t_{k} y\right)-t_{k} y \nu\left(c_{k}\right)}{c_{k}} \stackrel{D}{\longrightarrow} N(0, y), \quad \text { for each } y>0 .
$$

However, the centring here can be deleted, because

$$
\frac{t_{k}\left|\nu\left(c_{k}\right)\right|}{c_{k}}=\left|\frac{t_{k} A\left(c_{k}\right)}{c_{k}}-t_{k} D\left(c_{k}\right)\right| \leqslant \frac{t_{k}\left|A\left(c_{k}\right)\right|}{c_{k}}+t_{k} \bar{\Pi}\left(c_{k}\right),
$$

while

$$
\begin{aligned}
\frac{t_{k}\left|A\left(c_{k}\right)\right|}{c_{k}} & \leqslant \mathrm{o}\left(\frac{t_{k}\left|A\left(x_{k}\right)\right|}{x_{k}}\right)+\frac{t_{k}\left|\int_{x_{k}}^{c_{k}} D(y) d y\right|}{c_{k}} \\
& \leqslant \mathrm{o}(1)(\text { by }(2.39) \text { and }(2.38))+t_{k} \bar{\Pi}\left(x_{k}\right)=\mathrm{o}(1) .
\end{aligned}
$$


It follows that $X\left(t_{k} y\right) / c_{k} \stackrel{D}{\longrightarrow} N(0, y)$ for each $y>0$ and this can be extended to a functional version as in the proof of Proposition 3.

Conversely, if there is a $t_{k} \downarrow 0$ such that $X\left(t_{k}\right) / c_{k} \stackrel{D}{\longrightarrow} N(0,1)$, then we easily get (1.13), using [8, Theorem 2.5].

Proposition 5. Equation (1.13) implies that (1.15) has a.s. cluster set $\mathscr{K}$.

Proof. We modify the proof of Theorem 1 as follows. Let (1.13) hold, so $V(x)>0$ on a neighbourhood of 0 . This time, take

$$
\zeta_{k}:=\frac{x_{k}^{2} \bar{\Pi}\left(x_{k}\right)+x_{k}\left|A\left(x_{k}\right)\right|}{V\left(x_{k}\right)} \rightarrow 0, \quad \text { as } k \uparrow \infty, \quad \text { for some } x_{k} \downarrow 0 .
$$

We can assume $\zeta_{k} \downarrow 0$ and $\zeta_{k} \leqslant k^{-2 / \varepsilon}$, where $\varepsilon \in(0,1 / 32)$. Since $\bar{\Pi}(0+)>0$, we have $\zeta_{k}^{1 / 4} /\left(\bar{\Pi}\left(x_{k}\right)+\left|A\left(x_{k}\right)\right| / x_{k}\right) \rightarrow 0$ and can further assume

$$
\frac{\zeta_{k}^{1 / 4}}{\bar{\Pi}\left(x_{k}\right)+\left|A\left(x_{k}\right)\right| / x_{k}} \downarrow 0, \quad \text { as } k \uparrow \infty,
$$

and

$$
\frac{\zeta_{k}^{1 / 4}}{\bar{\Pi}\left(x_{k}\right)+\left|A\left(x_{k}\right)\right| / x_{k}}<\frac{1}{(k+1)^{2 / \varepsilon}} \overline{\bar{\Pi}\left(x_{k-1}\right)+\left|A\left(x_{k-1}\right)\right| / x_{k-1}} .
$$

Define $r_{k} \uparrow \infty$ so that

$$
\lambda^{r_{k}}=\frac{\zeta_{k}^{1 / 4}}{\bar{\Pi}\left(x_{k}\right)+\left|A\left(x_{k}\right)\right| / x_{k}},
$$

where $0<\lambda<1$, and define $b(t)$, depending on $\varepsilon$ but not on $\lambda$, by: $b(0)=0$ and

$$
b(t)=t^{1 / 2-\varepsilon}\left(\frac{\zeta_{k-1}^{1 / 4}}{\bar{\Pi}\left(x_{k-1}\right)+\left|A\left(x_{k}\right)\right| / x_{k}}\right)^{\varepsilon} \sqrt{2 V\left(x_{k-1}\right) \log (k+1)}
$$

(the $\log$ here is to the base $e$ ), whenever

$$
\frac{\zeta_{k}^{1 / 4}}{\bar{\Pi}\left(x_{k}\right)+\left|A\left(x_{k}\right)\right| / x_{k}}<t \leqslant \frac{\zeta_{k-1}^{1 / 4}}{\bar{\Pi}\left(x_{k-1}\right)+\left|A\left(x_{k-1}\right)\right| / x_{k-1}}, \quad k=1,2, \ldots .
$$

This $b(t)$ also satisfies (2.6) as stated. Now follow the proof of Proposition 1 from (2.6) on. In (2.7) and (2.9) we can replace $\bar{\Pi}\left(x_{k}\right)$ by $\bar{\Pi}\left(x_{k}\right)+\left|A\left(x_{k}\right)\right| / x_{k}$, so

$$
\sum_{k \geqslant 1} \sum_{r_{k-1} \leqslant r<r_{k}} \lambda^{r}\left(\bar{\Pi}\left(x_{k-1}\right)+\left|A\left(x_{k-1}\right)\right| / x_{k-1}\right)<\infty,
$$

and

$$
\lim _{k \rightarrow \infty} \max _{r_{k-1} \leqslant r<r_{k}} \lambda^{r}\left(\bar{\Pi}\left(x_{k-1}\right)+\left|A\left(x_{k-1}\right)\right| / x_{k-1}\right)=0,
$$

and we still have (2.8). Continuing, we get (2.12) and (2.13), as stated, as before (note that (2.16) follows from (2.41) when $\bar{\Pi}\left(x_{k-1}\right)$ is replaced by $\bar{\Pi}\left(x_{k-1}\right)+\left|A\left(x_{k-1}\right)\right| / x_{k-1}$, and (2.17) holds in the new setup). At this stage we can modify (2.13) to

$$
\sum_{r \geqslant 1} P\left\{\left|X_{p}\left(y \lambda^{r}\right)\right|>a y^{1 / 2} b\left(\lambda^{r}\right)\right\}<\infty \quad \text { for all } a>1 .
$$


To deduce this from (2.13), it suffices that $\lambda^{r}\left|\nu\left(b\left(\lambda^{r}\right)\right)\right|=\mathrm{o}\left(b\left(\lambda^{r}\right)\right)$, as $r \rightarrow \infty$, and, by (2.19), for this it suffices that

$$
\lim _{k \rightarrow \infty} \sup _{\lambda^{r_{k}}<t \leqslant \lambda^{r_{k-1}}} \frac{t\left|\nu\left(x_{k-1}\right)\right|}{b(t)}=0 .
$$

Now for $\lambda^{r_{k}}<t \leqslant \lambda^{r_{k-1}}$ we have by (1.6) and (2.6)

$$
\begin{aligned}
\frac{t\left|\nu\left(x_{k-1}\right)\right|}{b(t)} & \leqslant \frac{\lambda^{r_{k-1}}\left(x_{k-1} \bar{\Pi}\left(x_{k-1}\right)+\left|A\left(x_{k-1}\right)\right|\right)}{\lambda^{r_{k-1} / 2} \sqrt{2 V\left(x_{k-1}\right) \log (k+1)}}=\frac{\lambda^{r_{k-1} / 2} \zeta_{k-1} \sqrt{V\left(x_{k-1}\right)}}{x_{k-1} \sqrt{2} \log (k+1)} \\
& =\frac{\zeta_{k-1}^{9 / 8}}{\sqrt{2 \log (k+1)}} \sqrt{\frac{V\left(x_{k-1}\right)}{x_{k-1}^{2} \bar{\Pi}\left(x_{k-1}\right)+x_{k-1}\left|A\left(x_{k-1}\right)\right|}}=\frac{\zeta_{k-1}^{1 / 8}}{\sqrt{2 \log (k+1)}} \rightarrow 0 .
\end{aligned}
$$

Thus indeed (2.43) holds. We can then follow the rest of the proof of (1.9) in Theorem 1, omitting the centring term $\nu(\cdot)$ in Lemmas 2, 3, and 4, and through to the end of this part of the proof, just prior to Proposition 2. This establishes that (1.15) has a.s. accumulation points $\mathscr{K}$.

Proof of Theorem 3. Assume (ii) of Theorem 3, so (1.17) or (1.18) holds (we will use the version with $V(\cdot)$ and $\nu(\cdot)$, rather than $U(\cdot)$ and $A(\cdot))$. Suppose first that (1.17) fails, so

$$
V(x) \leqslant a x^{2} \bar{\Pi}(x) \text { for all } x \leqslant \text { some } x_{0}, \text { for some } a>0 \text {; }
$$

then (1.18) must hold. Thus we can take a sequence $x_{k} \downarrow 0$ such that $\left|\nu\left(x_{k}\right)\right|>0$. Hence we can assume $\nu\left(x_{k}\right)>0$ for all $k$, or $\nu\left(x_{k}\right)<0$ for all $k$. Let us assume that $\nu\left(x_{k}\right)>0$ for all $k$, the other case being handled similarly. Then we have

$$
\zeta_{k}:=\frac{x_{k} \bar{\Pi}\left(x_{k}\right)}{\nu\left(x_{k}\right)} \downarrow 0 \quad(k \rightarrow \infty) .
$$

It is always true that $\lim _{x \downarrow 0} x \nu(x)=0$, so by taking a subsequence if necessary, we can assume that $\zeta_{k} / \zeta_{k-1} \downarrow 0, \zeta_{k} / \bar{\Pi}\left(x_{k}\right) \downarrow 0$, and $x_{k} \nu\left(x_{k}\right) \downarrow 0$ as $k \rightarrow \infty$, and that $\zeta_{k} \leqslant(k+1)^{-2}$. Given $0<\lambda<1$, define

$$
r_{k}=\log _{\lambda}\left(\frac{x_{k}}{\nu\left(x_{k}\right)}\right), \quad \text { so that } \lambda^{r_{k}}=\frac{x_{k}}{\nu\left(x_{k}\right)}=\frac{\zeta_{k}}{\bar{\Pi}\left(x_{k}\right)} \downarrow 0 \text { as } k \rightarrow \infty .
$$

Then $r_{k} \uparrow \infty$ and $r_{k}-r_{k-1} \rightarrow \infty$ as $k \rightarrow \infty$. Now fix $\varepsilon \in(1 / 2,1)$ and define $b(t)$ by

$$
b(t)=t^{1-\varepsilon} \lambda^{\varepsilon r_{k-1}} \nu\left(x_{k-1}\right) \text { when } \lambda^{r_{k}}<t \leqslant \lambda^{r_{k-1}} .
$$

We show that $b(t) / t^{1-\varepsilon}$ is nondecreasing. This is obvious except for $t$ at jump points, so consider

$$
\frac{b\left(\lambda^{r_{k}}+\right)}{b\left(\lambda^{r_{k}}\right)}=\frac{\lambda^{r_{k-1}} \nu\left(x_{k-1}\right)}{\lambda^{r_{k}} \nu\left(x_{k}\right)}
$$

We can assume that $x_{k}$ is chosen so that $\nu\left(x_{k}\right)$ converges monotonically to a limit $L$. If $0<L<\infty$, then equation (2.47) gives $b\left(\lambda^{r_{k}}+\right)>b\left(\lambda^{r_{k}}\right)$ for large $k$, since $r_{k}-r_{k-1} \rightarrow \infty$. The same is true if $L=0$ since then $\nu\left(x_{k}\right) \downarrow 0$ as $x_{k} \downarrow 0$. Hence suppose $L=\infty$. Then $\nu\left(x_{k}\right) \uparrow \infty$ as $x_{k} \downarrow 0$. Substituting (2.45) in (2.47) gives

$$
\frac{b\left(\lambda^{r_{k}}+\right)}{b\left(\lambda^{r_{k}}\right)}=\left(\frac{x_{k-1} \nu\left(x_{k}\right)}{x_{k} \nu\left(x_{k-1}\right)}\right)^{\varepsilon}\left(\frac{\nu\left(x_{k-1}\right)}{\nu\left(x_{k}\right)}\right)=\left(\frac{x_{k-1}}{x_{k}}\right)^{\varepsilon}\left(\frac{\nu\left(x_{k-1}\right)}{\nu\left(x_{k}\right)}\right)^{1-\varepsilon} .
$$

Since $1 / 2<\varepsilon<1$, we have $1-\varepsilon<1 / 2<\varepsilon$, and $\nu\left(x_{k-1}\right) \leqslant \nu\left(x_{k}\right)$, so the last expression is not smaller than

$$
\left(\frac{x_{k-1} \nu\left(x_{k-1}\right)}{x_{k} \nu\left(x_{k}\right)}\right)^{\varepsilon} \geqslant 1
$$

(because we made $x_{k} \nu\left(x_{k}\right) \downarrow 0$ as $\left.x_{k} \downarrow 0\right)$. Thus $b(t) / t^{1-\varepsilon}$ is nondecreasing. 
Next note that

$$
\sum_{k} \sum_{r_{k-1}<r \leqslant r_{k-1}} \lambda^{r} \bar{\Pi}\left(x_{k-1}\right) \leqslant 2 \sum_{k} \lambda^{r_{k-1}} \bar{\Pi}\left(x_{k-1}\right) \leqslant 2 \sum_{k} \zeta_{k-1} \leqslant 2 \sum_{k} k^{-2}
$$

is finite. Use (2.1) to write

$$
X(t)=t \nu\left(x_{k-1}\right)+\sigma B(t)+X^{(1)}\left(t, x_{k-1}\right)+X^{(2)}\left(t, x_{k-1}\right),
$$

where, as in (2.15) and (2.16),

$$
\sum_{k} P\left\{\sup _{0 \leqslant s \leqslant \lambda^{r_{k-1}}}\left|X^{(2)}\left(s, x_{k-1}\right)\right|>0\right\} \leqslant \sum_{k}\left(1-e^{-\lambda^{r_{k-1}} \bar{\Pi}\left(x_{k-1}\right)}\right)<\infty .
$$

By Chebychev and (2.2), for $y>0$

$$
P\left\{\left|\sigma B\left(\lambda^{r_{k-1}}\right)+X^{(1)}\left(\lambda^{r_{k-1}}, x_{k-1}\right)\right|>y \sqrt{\lambda^{r_{k-1}} V\left(x_{k-1}\right)}\right\} \leqslant y^{-2} .
$$

Apply this with

$$
y=y_{k}=\frac{\delta \lambda^{r_{k-1}} \nu\left(x_{k-1}\right)}{\sqrt{\lambda^{r_{k-1}} V\left(x_{k-1}\right)}}=\frac{\delta b\left(\lambda^{r_{k-1}}\right)}{\sqrt{\lambda^{r_{k-1}} V\left(x_{k-1}\right)}}, \quad \delta>0 .
$$

Note that

$$
\left(\delta^{-1} y_{k}\right)^{2}=\frac{\lambda^{r_{k-1}} \nu^{2}\left(x_{k-1}\right)}{V\left(x_{k-1}\right)}=\frac{x_{k-1} \nu\left(x_{k-1}\right)}{V\left(x_{k-1}\right)} \geqslant \frac{x_{k-1} \nu\left(x_{k-1}\right)}{a x_{k-1}^{2} \bar{\Pi}\left(x_{k-1}\right)}=\frac{\zeta_{k}^{-1}}{a} \rightarrow \infty,
$$

so by (2.48), (2.49), and (2.50), $X\left(\lambda^{r_{k-1}}\right) / b\left(\lambda^{r_{k-1}}\right) \stackrel{\mathrm{P}}{\longrightarrow} 1$, and hence

$$
\limsup _{t \downarrow 0} \frac{|X(t)|}{b(t)} \geqslant 1 \text { a.s. }
$$

Next use a variant of (2.23) to get

$$
\begin{aligned}
& P\left\{\sup _{0 \leqslant t \leqslant \lambda^{r_{k-1}}}\left|\sigma B(t)+X^{(1)}\left(t, x_{k}\right)\right|>\delta b\left(\lambda^{r_{k-1}}\right)\right\} \\
& \quad \leqslant 2 P\left\{\left|\sigma B\left(\lambda^{r_{k-1}}\right)+X^{(1)}\left(\lambda^{r_{k-1}}, x_{k}\right)\right|>(\delta / 2) b\left(\lambda^{r_{k-1}}\right)\right\},
\end{aligned}
$$

for large $k$; note that $b^{2}\left(\lambda^{r_{k-1}}\right) /\left(\lambda^{r_{k-1}} V\left(x_{k-1}\right)\right)=\left(y_{k} / \delta\right)^{2} \rightarrow \infty$. Hence

$$
\sum_{k} P\left\{\sup _{0 \leqslant t \leqslant \lambda^{r_{k-1}}}\left|\sigma B(t)+X^{(1)}\left(t, x_{k}\right)\right|>\delta b\left(\lambda^{r_{k-1}}\right)\right\} \leqslant 8 \delta^{-2} \sum_{k \geqslant 1} y_{k}^{-2}<\infty,
$$

and by (2.49) we can replace $\sigma B(t)+X^{(1)}\left(t, x_{k}\right)$ with $X(t)-t \nu\left(x_{k-1}\right)$ here. Thus

$$
\lim _{k \rightarrow \infty} \frac{\sup _{0 \leqslant t \leqslant \lambda^{r k-1}}\left|X(t)-t \nu\left(x_{k-1}\right)\right|}{b\left(\lambda^{r_{k-1}}\right)}=0 \text { a.s. }
$$

By (2.46) this means

$$
\limsup _{k \rightarrow \infty}\left(\frac{\sup _{0 \leqslant t \leqslant \lambda^{r_{k-1}}}|X(t)|}{b\left(\lambda^{r_{k-1}}\right)}\right) \leqslant 1 \text { a.s. }
$$

Given $t>0$ choose $k=k(t)$ so that $\lambda^{r_{k}}<t \leqslant \lambda^{r_{k-1}}$. Then $b(t)=b\left(\lambda^{r_{k-1}}\right)$, and

$$
\limsup _{t \downarrow 0}\left(\frac{|X(t)|}{b(t)}\right) \leqslant \limsup _{k \rightarrow \infty}\left(\frac{\sup _{0 \leqslant t \leqslant \lambda^{r} k-1}|X(t)|}{b\left(\lambda^{r_{k-1}}\right)}\right) \leqslant 1 \text { a.s. }
$$

Together with (2.51) this gives (1.16).

Next assume (1.17), so (1.8) holds. Define $x_{k} \downarrow 0, \zeta_{k} \downarrow 0$, and $r_{k} \rightarrow \infty$ by (2.3) and (2.5) in the proof of Proposition 1, and we still have (2.7) of that proof. Furthermore, (2.12) holds 
again, for the same $I_{k}=\left\{r \in \mathbb{N}: r_{k-1} \leqslant r \leqslant r_{k-1}+\varepsilon^{-1}\left|\log _{\lambda} k\right|\right\}$. With $p=1=c$. in (2.12), we have for $y>0$

$$
\sum_{k} \sum_{r \in I_{k}} \sup _{x}\left|P\left\{X\left(y \lambda^{r}\right)-y \lambda^{r} \nu\left(x_{k-1}\right) \leqslant x \sqrt{y \lambda^{r} V\left(x_{k-1}\right)}\right\}-\Phi(x)\right|<\infty .
$$

Suppose first that

$$
y_{k}:=\frac{\lambda^{r_{k-1}} \nu\left(x_{k-1}\right)}{\sqrt{\lambda^{r_{k-1}} V\left(x_{k-1}\right)}}
$$

tends to $+\infty$ or $-\infty$ through a subsequence. Suppose it were $+\infty$; then by taking a further subsequence assume $y_{k} / \log _{2} k \rightarrow \infty$. Define $b(t)$ by $(2.46)$. Then $b\left(\lambda^{r_{k-1}}\right)=y_{k} \sqrt{\lambda^{r_{k-1}} V\left(x_{k-1}\right)}=$ $\lambda^{r_{k-1}} \nu\left(x_{k-1}\right)$, and by $(2.53)$ (with $r=r_{k-1}, x=\delta y_{k}, \delta>0, y=1$ )

$$
\sum_{k} P\left\{\left|X\left(\lambda^{r_{k-1}}\right)-b\left(\lambda^{r_{k-1}}\right)\right|>\delta b\left(\lambda^{r_{k-1}}\right)\right\} \leqslant 2 \sum_{k}\left(1-\Phi\left(\delta y_{k}\right)\right)<\infty .
$$

Repeating the proof from (2.50) on, we again arrive at (1.16).

Alternatively, $y_{k}$ in (2.54) is bounded. Then for $r_{k-1} \leqslant r$,

$$
\frac{\lambda^{r} \nu\left(x_{k-1}\right)}{\sqrt{\lambda^{r} V\left(x_{k-1}\right)}}=\sqrt{\lambda^{r-r_{k-1}}} \frac{\lambda^{r_{k-1}} \nu\left(x_{k-1}\right)}{\sqrt{\lambda^{r_{k-1}} V\left(x_{k-1}\right)}} .
$$

is bounded, and (2.53) gives, for $y>0$

$$
\sum_{k} \sum_{r \in I_{k}} \sup _{x}\left|P\left\{X\left(y \lambda^{r}\right) \leqslant(x+\mathrm{O}(1)) \sqrt{y \lambda^{r} V\left(x_{k-1}\right)}\right\}-\Phi(x)\right|<\infty .
$$

This time define $b(t)$ as in (2.6) in the proof of Proposition 1. Then follow exactly that proof, with (2.55) replacing (2.12) (we ultimately take $x=x(k, r)>a \sqrt{2 \log k}$ in the proof of Proposition 1, so the $\mathrm{O}(1)$ in (2.55) is irrelevant), and discarding the centring throughout, to get (1.9) without the centring, and this implies (1.16).

Conversely, assume (1.16), and assume that (ii) fails. Then neither (1.17) nor (1.18) holds. In the reverse direction of Theorem 1 we have (2.29) with $a(t)=0$, and so (2.30) holds. Thus

$$
\limsup _{t \downarrow 0} \frac{t|\nu(b(t))|}{b(t)}=1,
$$

and also $t \bar{\Pi}(b(t)) \rightarrow 0$ by [8, of Lemma 5.1]. However, since (1.18) fails

$$
\frac{t \nu(b(t))}{b(t)}=\mathrm{O}(t \bar{\Pi}(b(t))) \rightarrow 0
$$

and the contradiction completes the proof.

That (ii) and (iii) in Theorem 3 are equivalent is obvious, and (iv) obviously implies (ii). Finally, let (ii) hold. If (iv) fails then

$$
U(x)=\mathrm{O}\left(x|A(x)|+x^{2} \bar{\Pi}(x)\right) \quad \text { and } \quad x|A(x)|=\mathrm{O}(U(x)) .
$$

Suppose $U(x)=\mathrm{O}\left(x^{2} \bar{\Pi}(x)\right)$, then $x|A(x)|=\mathrm{O}\left(x^{2} \bar{\Pi}(x)\right)$, contradicting (1.18). Alternatively, suppose $|A(x)|=\mathrm{O}(x \bar{\Pi}(x))$, then $U(x)=\mathrm{O}\left(x^{2} \bar{\Pi}(x)\right)$, contradicting (1.17). Thus (ii) implies (iv).

Proof of Theorem 4. Assume $\bar{\Pi}(0+)>0$ and let (1.23) hold. Choose $x_{k} \downarrow 0$ such that $\left|A\left(x_{k}\right)\right|>0$, thus $A\left(x_{k}\right)>0$ (say), and, as $k \rightarrow \infty$,

$$
\frac{A\left(x_{k}\right)}{\sqrt{\bar{\Pi}\left(x_{k}\right) U\left(x_{k}\right)}} \rightarrow \infty .
$$


Before proceeding further we show that this implies $\sigma^{2}=0$. Suppose by way of contradiction that $\sigma^{2}>0$. Then from (1.4), (1.6), and (2.56)

$$
\frac{A\left(x_{k}\right)}{\sqrt{\bar{\Pi}\left(x_{k}\right)}}=\frac{\nu\left(x_{k}\right)+x_{k} D\left(x_{k}\right)}{\sqrt{\bar{\Pi}\left(x_{k}\right)}}=\frac{\gamma-\int_{x_{k}}^{1} y D(d y)+x_{k} D\left(x_{k}\right)}{\sqrt{\bar{\Pi}\left(x_{k}\right)}} \rightarrow \infty .
$$

Since $\bar{\Pi}(0+)>0$ and $\left|x_{k} D\left(x_{k}\right)\right| / \sqrt{\bar{\Pi}\left(x_{k}\right)} \leqslant \sqrt{x_{k}^{2} \bar{\Pi}\left(x_{k}\right)} \rightarrow 0$, we have

$$
\frac{-\int_{x_{k}}^{1} y D(d y)}{\sqrt{\bar{\Pi}\left(x_{k}\right)}} \rightarrow \infty \text {. }
$$

However, using the Cauchy-Schwarz inequality we deduce that

$$
\begin{aligned}
\frac{\left(\int_{x_{k}<|y| \leqslant 1} y D(d y)\right)^{2}}{\bar{\Pi}\left(x_{k}\right)} & \leqslant \frac{\int_{x_{k}<|y| \leqslant 1} y^{2} \Pi(d y) \int_{x_{k}<|y| \leqslant 1} \Pi(d y)}{\bar{\Pi}\left(x_{k}\right)} \\
& =\int_{x_{k}<|y| \leqslant 1} y^{2} \Pi(d y) \frac{\left(\bar{\Pi}\left(x_{k}\right)-\bar{\Pi}(1)\right)}{\bar{\Pi}\left(x_{k}\right)} \\
& \leqslant \int_{0<|y| \leqslant 1} y^{2} \Pi(d y)<\infty .
\end{aligned}
$$

This contradiction shows that $\sigma^{2}=0$.

Now define

$$
t_{k}=\frac{1}{A\left(x_{k}\right)} \sqrt{\frac{U\left(x_{k}\right)}{\bar{\Pi}\left(x_{k}\right)}} .
$$

Then

$$
t_{k} \bar{\Pi}\left(x_{k}\right)=\frac{\sqrt{U\left(x_{k}\right) \bar{\Pi}\left(x_{k}\right)}}{A\left(x_{k}\right)} \rightarrow 0
$$

and so since $\bar{\Pi}(0+)>0, t_{k} \rightarrow 0$. Also

$$
\frac{U\left(x_{k}\right)}{t_{k} A^{2}\left(x_{k}\right)}=\frac{1}{A\left(x_{k}\right)} \sqrt{\bar{\Pi}\left(x_{k}\right) U\left(x_{k}\right)} \rightarrow 0 .
$$

Define $b_{k}=t_{k} A\left(x_{k}\right)$; then

$$
\frac{b_{k}}{x_{k}}=\frac{t_{k} A\left(x_{k}\right)}{x_{k}}=\sqrt{\frac{U\left(x_{k}\right)}{x_{k}^{2} \bar{\Pi}\left(x_{k}\right)}} \geqslant 1 .
$$

Now since $b_{k} \geqslant x_{k}$

$$
\begin{aligned}
\frac{t_{k} U\left(b_{k}\right)}{b_{k}^{2}} & =\frac{U\left(x_{k}\right)}{t_{k} A^{2}\left(x_{k}\right)}+\frac{2 t_{k} \int_{x_{k}}^{b_{k}} y \bar{\Pi}(y) d y}{b_{k}^{2}} \quad\left(\text { recall } \sigma^{2}=0\right) \\
& \leqslant \mathrm{o}(1)+t_{k} \bar{\Pi}\left(x_{k}\right)=\mathrm{o}(1) \quad(\text { by }(2.58) \text { and }(2.57)) .
\end{aligned}
$$

Since $U(x) \geqslant x^{2} \bar{\Pi}(x)$, this means

$$
\lim _{k \rightarrow \infty} t_{k} \bar{\Pi}\left(\varepsilon b_{k}\right)=0 \quad \text { for all } \varepsilon>0 .
$$

Next, since $b_{k} \geqslant x_{k}$,

$$
\frac{t_{k} A\left(b_{k}\right)}{b_{k}}=1+\frac{t_{k} \int_{x_{k}}^{b_{k}}\left(\bar{\Pi}^{+}(y)-\bar{\Pi}^{-}(y)\right) d y}{b_{k}}=1+\mathrm{O}\left(t_{k} \bar{\Pi}\left(x_{k}\right)\right)=1+\mathrm{o}(1) .
$$


Use (2.1) and (1.6) to write

$$
X\left(t_{k}\right)=t_{k}\left(A\left(b_{k}\right)-b_{k} D\left(b_{k}\right)\right)+X^{(1)}\left(t_{k}, b_{k}\right)+X^{(2)}\left(t_{k}, b_{k}\right),
$$

where the Brownian term is absent since $\sigma^{2}=0$. Because of (2.60) and (2.15) we can ignore the big jump contribution to $X\left(t_{k}\right)$. Next, $t_{k} b_{k}\left|D\left(b_{k}\right)\right| \leqslant t_{k} b_{k} \bar{\Pi}\left(b_{k}\right)=\mathrm{o}\left(b_{k}\right)$. Now, $X^{(1)}\left(t_{k}, b_{k}\right)$ has expectation 0 and variance

$$
t_{k} \int_{|x| \leqslant b_{k}} y^{2} \Pi(d y) \leqslant t_{k} U\left(b_{k}\right)=\mathrm{o}\left(b_{k}^{2}\right) \quad(\text { by }(2.59)) ;
$$

so by Chebychev's inequality, $X^{(1)}\left(t_{k}, b_{k}\right)=o_{P}\left(b_{k}\right)$. Then (1.22) follows from

$$
\frac{X\left(t_{k}\right)}{b_{k}}=\frac{t_{k} A\left(b_{k}\right)}{b_{k}}+\mathrm{o}_{P}(1)=1+\mathrm{o}_{P}(1) \quad(\text { by }(2.61))
$$

Conversely, suppose $X\left(t_{k}\right) / b_{k} \stackrel{\mathrm{P}}{\longrightarrow} 1$. From [8, Lemma 5.1] with $t=t_{k}, a\left(t_{k}\right)=b_{k}$, we get (2.60), and in the decomposition (2.62) we can ignore the terms $X^{(2)}\left(t_{k}, b_{k}\right)$ and $t_{k} D\left(b_{k}\right)$ as before. Thus, defining

$$
\widehat{X}\left(t_{k}\right):=t_{k} A\left(b_{k}\right)+\sigma B\left(t_{k}\right)+X^{(1)}\left(t_{k}, b_{k}\right),
$$

we have $\widehat{X}\left(t_{k}\right) / b_{k} \stackrel{\mathrm{P}}{\longrightarrow} 1$. However, $E e^{i \theta \widehat{X}\left(t_{k}\right)}=e^{-t_{k} \widehat{\Psi}(\theta)}$, where for $\theta \in \mathbb{R}$

$$
\widehat{\Psi}(\theta)=-i A\left(b_{k}\right) \theta+\frac{\sigma^{2} \theta^{2}}{2}+\int_{|x| \leqslant b_{k}}\left(1-e^{i \theta x}+i \theta x\right) \Pi(d x) .
$$

We must have $t_{k} \operatorname{Re} \widehat{\Psi}\left(\theta / b_{k}\right) \rightarrow 0$ for all $\theta \in \mathbb{R}$; thus for $|\theta| \leqslant 1$

$$
\begin{aligned}
\mathrm{o}(1) & =\frac{t_{k} \sigma^{2} \theta^{2}}{2 b_{k}^{2}}+t_{k} \int_{|x| \leqslant b_{k}}\left(1-\cos \left(\theta x / b_{k}\right)\right) \Pi(d x) \\
& \geqslant \frac{t_{k} \theta^{2}}{4 b_{k}^{2}}\left(\sigma^{2}+\int_{|x| \leqslant b_{k}} x^{2} \Pi(d x)\right)=\frac{t_{k} \theta^{2} V\left(b_{k}\right)}{4 b_{k}^{2}} .
\end{aligned}
$$

Also, $t_{k} \operatorname{Im} \widehat{\Psi}\left(\theta / b_{k}\right) \rightarrow-\theta$ for all $\theta \in \mathbb{R}$; thus for $|\theta| \leqslant 1$

$$
\begin{aligned}
\theta+\mathrm{o}(1) & =\frac{t_{k} A\left(b_{k}\right) \theta}{b_{k}}+t_{k} \int_{|x| \leqslant b_{k}}\left(\sin \left(\theta x / b_{k}\right)-\theta x / b_{k}\right) \Pi(d x) \\
& =\frac{t_{k} A\left(b_{k}\right) \theta}{b_{k}}+\mathrm{O}\left(\frac{t_{k}}{b_{k}^{2}} \int_{|x| \leqslant b_{k}} x^{2} \Pi(d x)\right)=\frac{t_{k} A\left(b_{k}\right) \theta}{b_{k}}+\mathrm{O}\left(\frac{t_{k} V\left(b_{k}\right)}{b_{k}^{2}}\right) .
\end{aligned}
$$

Thus $t_{k} A\left(b_{k}\right) / b_{k} \rightarrow 1$. But then

$$
\frac{A\left(b_{k}\right)}{\sqrt{U\left(b_{k}\right) \bar{\Pi}\left(b_{k}\right)}}=\frac{t_{k} A\left(b_{k}\right)}{b_{k}} \sqrt{\left(\frac{b_{k}^{2}}{t_{k} U\left(b_{k}\right)}\right)\left(\frac{1}{t_{k} \bar{\Pi}\left(b_{k}\right)}\right)} \rightarrow \infty .
$$

Hence (1.23) holds, and so $\sigma^{2}=0$ again.

Finally let (1.23) hold. Then we get (1.21) from (2.59) and (2.61), and conversely (1.21) implies (1.23) because $U(x) \geqslant x^{2} \bar{\Pi}(x)$.

Remarks. When $\Pi(\cdot) \equiv 0$, equation (1.22) is equivalent to $\sigma^{2}=0$ and $\gamma \neq 0$; because these imply $X(t) /(t|\gamma|)= \pm 1$, certainly $X(t) /(t|\gamma|) \stackrel{\mathrm{P}}{\longrightarrow} \pm 1$. Conversely, if $\Pi(\cdot) \equiv 0$ and there are $t_{k} \downarrow 0, b_{k} \rightarrow 0$, such that $X\left(t_{k}\right) / b_{k} \stackrel{\mathrm{P}}{\longrightarrow} \pm 1$, then

$$
E e^{i \theta\left(\gamma t_{k}+\sigma B\left(t_{k}\right)\right) / b_{k}}=e^{i \theta \gamma t_{k} / b_{k}-1 / 2 \sigma^{2} \theta^{2} t_{k} / b_{k}^{2}} \rightarrow e^{ \pm i \theta},
$$

so $\gamma t_{k} / b_{k} \rightarrow \pm 1$ and $\sigma^{2} t_{k} / b_{k}^{2} \rightarrow 0$. The first of these implies $\gamma \neq 0$, so $t_{k} / b_{k} \rightarrow 1 /|\gamma|$, and thus $t_{k} / b_{k}^{2} \rightarrow \infty$; then the second gives $\sigma^{2}=0$. 
Proof of Theorem 5. Now $[X]_{t}$ is a subordinator with characteristic triplet $\left(\gamma_{[X]}, 0, \Pi_{[X]}\right)$, where

$$
\gamma_{[X]}=\sigma^{2}+\int_{(0,1]} x \Pi_{[X]}(d x) \quad \text { and } \quad \bar{\Pi}_{[X]}(x)=\bar{\Pi}(\sqrt{x}), \quad x>0 .
$$

Hence we can calculate, in an obvious notation,

$$
A_{[X]}(x)=\gamma_{[X]}+x \bar{\Pi}_{[X]}(x)-\int_{(x, 1]} y \Pi_{[X]}(d y)=\sigma^{2}+2 \int_{0}^{\sqrt{x}} y \bar{\Pi}(y) d y=U(\sqrt{x}),
$$

and

$$
U_{[X]}(x)=0+2 \int_{0}^{x} y \bar{\Pi}_{[X]}(y) d y=4 \int_{0}^{\sqrt{x}} y^{3} \bar{\Pi}(y) d y \in\left[x^{2} \bar{\Pi}(\sqrt{x}), 2 x U(\sqrt{x})\right] .
$$

Consequently

$$
\frac{A_{[X]}(x)}{x \bar{\Pi}_{[X]}(x)}=\frac{U(\sqrt{x})}{(\sqrt{x})^{2} \bar{\Pi}(\sqrt{x})},
$$

and also

$$
\frac{A_{[X]}(x)}{\sqrt{\bar{\Pi}_{[X]}(x) U_{[X]}(x)}} \geqslant \frac{U(\sqrt{x})}{\sqrt{2 \bar{\Pi}(\sqrt{x}) x U(\sqrt{x})}}=\sqrt{\frac{U(\sqrt{x})}{2(\sqrt{x})^{2} \bar{\Pi}(\sqrt{x})}} .
$$

Thus (1.8) (for $X$ ) implies (1.18) and (1.23) (for $[X]$ ), and hence (1.24) and (1.25).

Conversely,

$$
\frac{x A_{[X]}(x)}{U_{[X]}(x)} \leqslant \frac{x U(\sqrt{x})}{x^{2} \bar{\Pi}(\sqrt{x})}=\frac{U(\sqrt{x})}{(\sqrt{x})^{2} \bar{\Pi}(\sqrt{x})},
$$

so (1.21) (for $[X])$ implies (1.8) (for $X$ ).

Acknowledgements. I am grateful to Boris Buchmann, Harry Kesten, and Alex Szimayer for many helpful comments and suggestions at various stages. Thanks also to a referee whose careful reading and helpful suggestions materially improved the exposition, and to Brett Witty for excellent Tex typing.

\section{References}

1. P. BALDI, 'Large deviations and functional iterated logarithm laws for diffusion processes', Prob. Theor. Rel. Fields 71 (1986) 435-453.

2. J. Bertoin, Lévy processes, Cambridge Tracts in Mathematics 121 (Cambridge University Press, Cambridge, 1996).

3. J. Bertoin, R. A. Doney, and R. A. Maller, 'Passage of Lévy processes across power law boundaries at small times', Ann. Prob. 36 (2008) 160-197.

4. N. H. Bingham, 'Fluctuation theory in continuous time', Adv. Appl. Probab. 7 (1975) 705-766.

5. B. Buchmann, R. A. Maller, and A. Szimayer, 'An almost sure functional limit theorem at zero for a Lévy process normed by the square root function, and applications', Prob. Theor. Rel. Fields 142 (2008) 219-247.

6. L. Caramellino, 'Strassen's law of the iterated logarithm for diffusion processes for small time', Stoch. Proc. Appl. 74 (1998) 1-19.

7. R. A. Doney, Fluctuation theory for Lévy processes, Ecole d'Eté de Probabilités de Saint-Flour XXXV2005, Lecture Notes in Mathematics (Springer, Berlin, 2007).

8. R. A. Doney and R. A. Maller, 'Stability and attraction to normality for Lévy processes at zero and infinity'. J. Theoret. Probab. 15 (2002) 751-792.

9. R. A. Doney and R. A. Maller, 'Moments of passage times for Lévy processes', Ann. Inst. Henri Poincaré 40 (2004) 279-297.

10. H. Finkelstein, 'The law of the iterated logarithm for empirical distributions', Ann. Math. Statist. 42 (1971) 607-615.

11. N. Gantert, 'An inversion of Strassen's law of the iterated logarithm for small time', Ann. Probab. 21 (1993) 1045-1049. 
12. H. Kesten, 'Sums of independent random variables-without moment conditions', Ann. Math. Statist. 43 (1972) 701-732.

13. A. Ya Khintchine, 'Sur la croissance locale des processes stochastiques homogènes à acroissements indépendants', Izv. Ahad. Nauk SSSR 3 (1939) 487-508.

14. P. LÉvy, Théorie de l'Addition des Variables Aléatoires (Gauthier-Villars, Paris, 1937).

15. V. V. Petrov, Sums of independent random variables (Springer, Berlin, 1975).

16. K. Sato, Lévy processes and infinitely divisible distributions (Cambridge University Press, Cambridge, 1999).

17. M. SAvov, 'Small time two-sided LIL behavior for Lévy processes at zero', Preprint, University of Manchester, 2008.

18. V. Strassen, 'An invariance principle for the law of the iterated logarithm', Z. Wahrscheinlichkeitstheorie und Verw. Gebiete 3 (1964) 211-226.

R. A. Maller

Centre for Mathematics and its Applications

Australian National University

Canberra, ACT 0200

Australia

and

School of Finance and Applied Statistics

Australian National University

Canberra, ACT 0200

Australia

Ross.Maller@anu.edu.au 